\title{
Efficient Method for Estimating Directions- of-Arrival of Partially Polarized Signals with Electromagnetic Vector Sensors
}

\author{
Kwok-Chiang Ho, Kah-Chye Tan, and B. T. G. Tan
}

\begin{abstract}
We have developed a high-resolution ESPRIT-based method for estimating the directions-of-arrival of partially polarized signals with electromagnetic vector sensors, each of which provides measurements of the complete electric and magnetic fields induced by electromagnetic signals. The method is computationally efficient since unlike many high-resolution methods, it does not involve searching across a multidimensional array manifold. In addition, the method has two variants, of which one is applicable to scenarios where $a$ priori information about the array system, such as the sensor positions, is unavailable.
\end{abstract}

\section{INTRODUCTION}

$\mathbf{I}$ N THE RECENT literature, there have been several studies on direction-of-arrival (DOA) estimation with electromagnetic (EM) vector sensors (VS's) that provide measurements of the complete electric and magnetic fields induced by EM signals [1]-[6]. Many advantages of using such VS's have been identified. Indeed, Tan et al. [2] (see also [3]-[5]) have established that with just one VS, one can uniquely determine the DOA's of two uncorrelated EM signals in general or three uncorrelated signals if they are skywaves. (One would need more than seven appropriately spaced scalar sensors, where each provides measurements of only one component of electric field, to determine uniquely the DOA's of three uncorrelated EM signals [8].) Via an explicit evaluation of the Cramér-Rao bound, Nehorai and Paldi [1] demonstrated that superior quality DOA estimates can be obtained with VS's. Therefore, DOA estimation systems employing VS's can be expected to be not only compact but also able to yield good performance.

Although much has been achieved in establishing the performance of EM VS's [1]-[5], the topic of DOA estimation methods customized for such sensors seems to have received little attention. The studies carried out by $\mathrm{Li}$ [6] and by Wong and Zoltowski [7] are among the few. On developing the methods proposed in [6] and [7], some specific relationships among the measurements of electric and magnetic fields, known as Maxwell's equations, were exploited. The methods are computationally efficient since unlike many high-

Manuscript received March 3, 1996; revised December 22, 1996. The associate editor coordinating the review of this paper and approving it for publication was Prof. Hagit Messer-Yaron.

K.-C. Ho and K.-C. Tan are with the Centre for Signal Processing, Nanyang Technological University, School of Electrical and Electronic Engineering, Singapore (e-mail: ekcho@.ntu.edu.sg).

B. T. G. Tan is with the Faculty of Science, National University of Singapore, Singapore.

Publisher Item Identifier S 1053-587X(97)07358-3. resolution methods, they do not involve searching across a multidimensional array manifold. Moreover, the method devised by $\mathrm{Li}$ [6] does not require a priori information about the array system such as the sensor positions.

The ESPRIT-based methods [6], [7] are designed for completely polarized (CP) signals. The polarization state of such a signal is constant, whereas that of a partially polarized (PP) or unpolarized (UP) signal varies with time. However, in some practical scenarios, PP or UP signals cannot be avoided. For example, even with a well-designed vertical-polarization Radar antenna, the signals radiated off the main beam axis will contain some varying amount of horizontally polarized component [9]. As the transmitting Radar rotates itself to scan over the desired sector, the polarization of signals received at an observation point varies with time. In addition, UP signals are sometimes deliberately generated. Indeed, one way of maximizing the use of allocated communication bandwidth is to transmit (and receive) simultaneously two independent messages, each of which utilizes the entire bandwidth allocated with the use of a single VS. This can be accomplished via exploitation of the two spatial degrees of freedom available in a transverse EM wave [1]. Consequently, the signals transmitted can even be UP. An efficient method for estimation of DOA's of PP signals has been proposed by Li and Stoica [10]. The method proposed in [10] has been designed for use with a uniform linear array comprising cross dipoles (each of which provides measurements of the electric but not the magnetic field) and is applicable to cases where the incoming signals are known to lie in a 2-D plane containing the cross dipoles.

In this paper, we first study the applicability of Li's method [6] for estimation of the DOA's of PP signals. Subsequently, we develop an ESPRIT-based method for estimating the DOA's of PP signals (with UP signals being the special case) that may arrive from anywhere in a 3-D space with EM VS's. Our method is computationally efficient. In addition, it has two variants of which one does not require a priori information about the sensor positions. Some of our results have been presented in [11], and here, we provide the detailed derivations of our results.

\section{DATA MODEL}

We shall adopt the data model proposed by Nehorai and Paldi [1] for an array of $m$ EM VS's receiving $n$ signals

$$
\mathbf{y}(t)=\sum_{k=1}^{n} \mathbf{e}\left(\phi_{k}, \psi_{k}\right) \otimes\left[\begin{array}{c}
\mathbf{I}_{3} \\
\left(\mathbf{u}_{k} \times\right)
\end{array}\right] \mathbf{V}_{k} \mathbf{x}_{k}(t)+\mathbf{n}(t)
$$


where $\mathbf{y}(t)$ and $\mathbf{n}(t)$ are $6 m \times 1$ complex vectors, respectively, given by

$$
\begin{aligned}
& \mathbf{y}(t)=\left[\mathbf{y}_{e}^{(1)}(t), \mathbf{y}_{h}^{(1)}(t), \cdots, \mathbf{y}_{e}^{(m)}(t), \mathbf{y}_{h}^{(m)}(t)\right]^{T} \\
& \in \mathbb{C}^{6 m \times 1} \\
& \mathbf{n}(t)=\left[\mathbf{n}_{e}^{(1)}(t), \mathbf{n}_{h}^{(1)}(t), \cdots, \mathbf{n}_{e}^{(m)}(t), \mathbf{n}_{h}^{(m)}(t)\right]^{T} \\
& \in \mathbb{C}^{6 m \times 1} \\
& \mathbf{e}\left(\phi_{k}, \psi_{k}\right)=\left[e^{-j \omega_{c} \tau_{1, k}}, \cdots, e^{-j \omega_{c} \tau_{m, k}}\right]^{T} \in \mathbb{C}^{m \times 1} \\
& \left(\mathbf{u}_{k} \times\right) \triangleq\left[\begin{array}{ccc}
0 & -u_{z}^{(k)} & u_{y}^{(k)} \\
u_{z}^{(k)} & 0 & -u_{x}^{(k)} \\
-u_{y}^{(k)} & u_{x}^{(k)} & 0
\end{array}\right] \\
& \mathbf{u}_{k}=\left[\begin{array}{l}
u_{x}^{(k)} \\
u_{y}^{(k)} \\
u_{z}^{(k)}
\end{array}\right]=\left[\begin{array}{c}
\cos \phi_{k} \cos \psi_{k} \\
\sin \phi_{k} \cos \psi_{k} \\
\sin \psi_{k}
\end{array}\right] \\
& \mathbf{V}_{k}=\left[\begin{array}{ll}
\mathbf{v}_{1, k} & \mathbf{v}_{2, k}
\end{array}\right] \\
& =\left[\begin{array}{cc}
-\sin \phi_{k} & -\cos \phi_{k} \sin \psi_{k} \\
\cos \phi_{k} & -\sin \phi_{k} \sin \psi_{k} \\
0 & \cos \psi_{k}
\end{array}\right]
\end{aligned}
$$

where

$\mathbf{I}_{l} \quad(l \times l)$ identity matrix;

$\otimes \quad$ Kronecker product operator;

" $T$ " transpose operator.

Note that $\mathbf{x}_{k}(t) \in \mathbb{C}^{2 \times 1}$ for $k=1, \cdots, n$, and $\mathbf{y}_{e}^{(l)}(t), \mathbf{y}_{h}^{(l)}(t)$, $\mathbf{n}_{e}^{(l)}(t), \mathbf{n}_{h}^{(l)}(t) \in \mathbb{C}^{1 \times 3}$ for $l=1, \cdots, m$.

It is worth mentioning the physical meaning associated with each of the above notations. For convenience, set up a Cartesian coordinate system with the origin being co-located with the reference sensor and each of the axes coinciding with a component of the electric field (and a component of magnetic field) measurable with the VS's. Then, $\mathbf{y}_{e}^{(l)}(t)$ and $-\mathbf{y}_{h}^{(l)}(t) / \eta$ are, respectively, the three-component measurements of the electric and magnetic fields at the $l$ th sensor at time $t$, where $\eta$ is the intrinsic impedance of the medium, and $\mathbf{n}_{e}^{(l)}(t)$ and $\mathbf{n}_{h}^{(l)}(t)$ are, correspondingly, the noise components in the measurements. Physically, the orientations of the three electric field components (and the magnetic field components) with respect to each VS can be unambiguously determined. For example, an EM VS can be constructed using three mutually perpendicular co-located short dipoles and three small loops. The three dipoles of every VS of an array have their axes parallel to the three coordinate axes, and the three loops have their normals being parallel to the coordinate axes (see [6]). Consequently, all VS's have exactly the same orientation. Since each dipole is short, the output voltage from each dipole is proportional to the electric field component induced along the dipole axis. Moreover, since each loop is small, the output voltage from each loop is proportional to the magnetic field component induced along the normal of the loop.

The two entries of the vector $\mathrm{x}_{k}(t)$ represent the complex envelopes of the $k$ th transmitted signals. The symbol $\omega_{c}$ denotes the frequency of the signals, $\phi_{k} \in(-\pi, \pi]$ and $\psi_{k} \in[-\pi / 2, \pi / 2]$ are, respectively, the azimuth and elevation of the $k$ th signal (see Fig. 1), $\tau_{l, k}$ is the differential delay of the $k$ th signal at the $l$ th sensor with respect to the reference

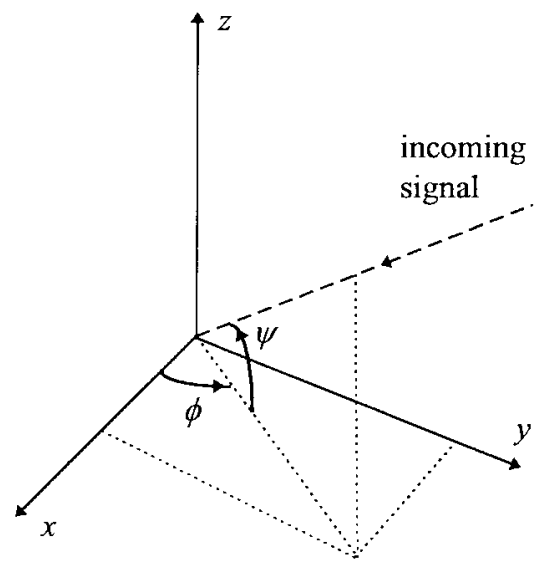

Fig. 1. DOA $(\phi, \psi)$ of an incoming signal.

sensor, the vector $\mathbf{u}_{k}$ is the unit vector pointing toward the DOA of the $k$ th signal, and the matrix $\left(\mathbf{u}_{k} \times\right)$ effects a crossproduct operation with $\mathbf{u}_{k}$. The vectors $\mathbf{v}_{1, k}$ and $\mathbf{v}_{2, k}$ are unit vectors that span the same plane as the electric and magnetic field vectors of the $k$ th signal.

The state of polarization of the $k$ th signal can be determined based on the covariance of the vector $\mathrm{x}_{k}(t)$. Indeed, the signal covariance matrix $E\left[\mathbf{x}_{k}(t) \mathbf{x}_{k}^{H}(t)\right]$ can be expressed as (see Lemma 1 of [12])

$$
E\left[\mathbf{x}_{k}(t) \mathbf{x}_{k}^{H}(t)\right]=\frac{\hat{\sigma}_{k}^{2}}{2} \mathbf{I}_{2}+\sigma_{k}^{2} \mathbf{Q}\left(\alpha_{k}\right) \mathbf{w}\left(\beta_{k}\right) \mathbf{w}^{H}\left(\beta_{k}\right) \mathbf{Q}^{H}\left(\alpha_{k}\right)
$$

where $\sigma_{k}^{2}, \hat{\sigma}_{k}^{2} \in \Re$

$$
\begin{aligned}
& \mathbf{Q}\left(\alpha_{k}\right)=\left(\begin{array}{cc}
\cos \alpha_{k} & \sin \alpha_{k} \\
-\sin \alpha_{k} & \cos \alpha_{k}
\end{array}\right) \\
& \mathbf{w}\left(\beta_{k}\right)=\left(\begin{array}{c}
\cos \beta_{k} \\
j \sin \beta_{k}
\end{array}\right)
\end{aligned}
$$

where $\alpha_{k} \in(-\pi / 2, \pi / 2]$ and $\beta_{k} \in[-\pi / 4, \pi / 4]$ are polarization parameters commonly referred to as the orientation angle and ellipticity angle, respectively, and " $E$ " and " $H$ " are, respectively, the expectation and Hermitian operators. The first term of the right-hand side of (5) corresponds to the UP component, and the second term corresponds to CP component, with powers $\hat{\sigma}_{k}^{2}$ and $\sigma_{k}^{2}$, respectively. The degree of polarization of the signal is defined as the ratio of the power of the $\mathrm{CP}$ component to the total power of the signal, i.e., $\sigma_{k}^{2} /\left(\hat{\sigma}_{k}^{2}+\sigma_{k}^{2}\right)$.

It can be easily verified that the signal covariance matrix $E\left[\mathrm{x}_{k}(t) \mathrm{x}_{k}^{H}(t)\right]$ as given by (5) is of rank 2 for the case of PP and UP signals (respectively, corresponding to nonzero $\sigma_{k}^{2}$ and $\hat{\sigma}_{k}^{2}$, and nonzero $\hat{\sigma}_{k}^{2}$ but $\sigma_{k}^{2}$ of zero value) and is rank deficient for the case of CP signals (corresponding to nonzero $\sigma_{k}^{2}$ but $\hat{\sigma}_{k}^{2}$ of zero value). Basically, a PP or UP signal exploits the two spatial degrees of freedom available in a transverse EM wave, whereas the CP signal utilizes only one. For convenience, the UP signal will be taken to be a special case of PP signal hereafter.

Equation (1) can be expressed in a more compact manner via matrix representation

$$
\mathbf{y}(t)=\mathbf{A} \mathbf{s}(t)+\mathbf{n}(t)
$$


where

$$
\begin{aligned}
\mathbf{A}= & {\left[\mathbf{e}\left(\phi_{1}, \psi_{1}\right) \otimes \mathbf{B}\left(\phi_{1}, \psi_{1}\right), \cdots, \mathbf{e}\left(\phi_{n}, \psi_{n}\right)\right.} \\
& \left.\otimes \mathbf{B}\left(\phi_{n}, \psi_{n}\right)\right] \in \mathbb{C}^{6 m \times 2 n} \\
\mathbf{s}(t)= & {\left[\mathbf{x}_{1}^{T}(t), \cdots, \mathbf{x}_{n}^{T}(t)\right]^{T} \in \mathbb{C}^{2 n \times 1} }
\end{aligned}
$$

and

$$
\mathbf{B}\left(\phi_{k}, \psi_{k}\right)=\left(\begin{array}{rr}
\mathbf{v}_{1, k} & \mathbf{v}_{2, k} \\
\mathbf{v}_{2, k} & -\mathbf{v}_{1, k}
\end{array}\right) \in \mathbb{C}^{6 \times 2}
$$

The set $\left\{\mathbf{B}\left(\phi_{k}, \psi_{k}\right): \phi_{k} \in(-\pi, \pi], \psi_{k} \in[-\pi / 2, \pi / 2]\right\}$ is the array manifold of UP signals associated with a single VS.

For CP signals, one can express $\mathbf{y}(t)$ in another way to avoid signal covariance matrices $E\left[\mathbf{x}_{k}(t) \mathbf{x}_{k}^{H}(t)\right]$ 's that are rank deficient

$$
\mathbf{y}(t)=\mathbf{A}^{(p)} \mathbf{s}^{(p)}(t)+\mathbf{n}(t)
$$

where

$$
\begin{aligned}
\mathbf{A}^{(p)}= & {\left[\mathbf{e}\left(\phi_{1}, \psi_{1}\right) \otimes \mathbf{B}\left(\phi_{1}, \psi_{1}\right) \mathbf{Q}\left(\alpha_{1}\right) \mathbf{w}\left(\beta_{1}\right), \cdots\right.} \\
& \left.\mathbf{e}\left(\phi_{n}, \psi_{n}\right) \otimes \mathbf{B}\left(\phi_{n}, \psi_{n}\right) \mathbf{Q}\left(\alpha_{n}\right) \mathbf{w}\left(\beta_{n}\right)\right] \\
\in & \mathbb{C}^{6 m \times n}
\end{aligned}
$$

and

$$
\mathbf{s}^{(p)}(t)=\left[s_{1}(t), \cdots, s_{n}(t)\right]^{T} \in \mathbb{C}^{n \times 1} .
$$

In this case, the signal covariance $E\left[s_{k}(t) s_{k}^{H}(t)\right]$ is simply the scalar $\sigma_{k}^{2}$ as it appears in (5), which yields the power of the $k$ th CP signal.

\section{RELEVANT WORK CARRIED OUT By LI}

It is beneficial to present a brief discussion of the method proposed by $\mathrm{Li}[6]$ since the underlying concepts and considerations motivate the development of our new method. The data model we adopt (see [1] for details) takes a form slightly different from Li's [6]. Our model is applicable to both $\mathrm{CP}$ signals and PP signals and thus provides the necessary framework for studying Li's method [6] for CP signals, as well as developing a new method for PP signals. For the case of CP signals, the two models are essentially equivalent.

Now, we shall discuss the essence of Li's method [6] in the light of our model. In fact, the relationships among $\mathbf{A}_{l}^{(p)}$, for $l=1, \cdots, 6$, where $\mathbf{A}_{l}^{(p)}$ is the $(m \times n)$ submatrix of $\mathbf{A}^{(p)}$ [as defined in (10)] consisting of the $l$ th, $(6+l)$ th, $\cdots$, $[6(m-1)+l]$ th rows of $\mathbf{A}^{(p)}$, which Li [6] has established, are crucial

$$
\left.\begin{array}{rl}
\mathbf{A}_{3}^{(p)}= & \mathbf{A}_{6}^{(p)} \mathbf{D}_{1}^{(p)} \\
\mathbf{A}_{1}^{(p)}+b_{k} \mathbf{A}_{4}^{(p)}=\left[\mathbf{A}_{3}^{(p)}+c_{k} \mathbf{A}_{6}^{(p)}\right] \mathbf{D}_{k}^{(p)} \\
\quad \text { for } k=2, \cdots, 5 \\
\mathbf{A}_{2}^{(p)}+b_{k} \mathbf{A}_{5}^{(p)}=\left[\mathbf{A}_{3}^{(p)}+c_{k} \mathbf{A}_{6}^{(p)}\right] \mathbf{D}_{k}^{(p)} \\
\quad \text { for } k=6, \cdots, 9
\end{array}\right\}
$$

where $b_{2}=b_{4}=b_{6}=b_{8}=c_{2}=c_{3}=c_{6}=c_{7}=1$, and $b_{3}=b_{5}=b_{7}=b_{9}=c_{4}=c_{5}=c_{8}=c_{9}=-1$. Physically, the $k$ th columns of $\mathbf{A}_{1}, \mathbf{A}_{2}$, and $\mathbf{A}_{3}$ are the steering vectors of the $k$ th signal associated with, respectively, the $x$ polarized, the $y$ polarized, and the $z$ polarized electric sensors. The $k$ th columns of $\mathbf{A}_{4}, \mathbf{A}_{5}$, and $\mathbf{A}_{6}$ are the steering vectors of the $k$ th signal associated with, respectively, the $x$ polarized, the $y$ polarized, and the $z$ polarized magnetic sensors. The matrices $\mathrm{D}_{k}^{(p)}$ 's are diagonal and of dimension $n \times n$. In addition, the diagonal elements of $\mathbf{D}_{k}^{(p)}$, for $k=1, \cdots, 9$, provide all the necessary information for determining uniquely the DOA's and polarizations of the signals. To estimate $\mathrm{D}_{k}^{(p)}$, s, Li [6] makes use of the eigenvectors of the array covariance matrix $E\left[\mathbf{y}(t) \mathbf{y}^{H}(t)\right]$ (which are referred to as $\mathbf{R}$ hereafter). Indeed, with the assumptions that conventional signal subspace methods (such as ESPRIT [13]) would require, in particular that

Assumption 1: the signals are uncorrelated;

Assumption 2: the signals and noise are uncorrelated;

Assumption 3: the noise covariance matrix is a constant multiple of identity matrix;

one can show that the matrices $\mathbf{A}^{(p)}$ and $\mathbf{E}^{(p)}$, which comprises the $n$ signal eigenvectors (i.e., the eigenvectors of $\mathbf{R}$ associated with the $n$ largest eigenvalues), satisfy the relationship

$$
\mathbf{E}^{(p)}=\mathbf{A}^{(p)} \mathbf{T}^{(p)}
$$

where $\mathbf{T}^{(p)} \in \mathbb{C}^{n \times n}$ is a unique nonsingular matrix. Now, let $\mathbf{E}_{l}^{(p)}$ be the $(m \times n)$ matrix that is extracted from $\mathbf{E}^{(p)}$ in the same way as $\mathbf{A}_{l}^{(p)}$ is extracted from $\mathbf{A}^{(p)}$ for $l=1, \cdots, 6$. Then, it follows from (11) and (12) that

$$
\left.\begin{array}{rl}
\mathbf{E}_{3}^{(p)}= & \mathbf{E}_{6}^{(p)} \Lambda_{1}^{(p)} \\
\mathbf{E}_{1}^{(p)}+b_{k} \mathbf{E}_{4}^{(p)}=\left[\mathbf{E}_{3}^{(p)}+c_{k} \mathbf{E}_{6}^{(p)}\right] \mathbf{\Lambda}_{k}^{(p)} \\
\\
\quad \text { for } k=2, \cdots, 5 \\
\mathbf{E}_{2}^{(p)}+b_{k} \mathbf{E}_{\tilde{5}}^{(p)}=\left[\mathbf{E}_{3}^{(p)}+c_{k} \mathbf{E}_{6}^{(p)}\right] \mathbf{\Lambda}_{k}^{(p)} \\
\text { for } k=6, \cdots, 9
\end{array}\right\}
$$

where

$$
\Lambda_{k}^{(p)}=\left[\mathbf{T}^{(p)}\right]^{-1} \mathbf{D}_{k}^{(p)} \mathbf{T}^{(p)} .
$$

The estimation process would then begin with the computation of $\mathbf{E}^{(p)}$ (and, thus, $\mathbf{E}_{l}^{(p)}$ for $l=1, \cdots, 6$ ). It is next followed by determining $\boldsymbol{\Lambda}_{k}^{(p)}$, for $k=1, \cdots, 9$, based on (13). From (14), the eigenvalues of $\Lambda_{k}^{(p)}$,s yield the diagonal elements of $\mathbf{D}_{k}^{(p)}$ 's. With the diagonal elements of $\mathbf{D}_{k}^{(p)}$ for $k=1, \cdots, 9$ being determined, one can obtain the DOA's and polarizations of the signals.

Remarks: 1) The fact that the measurements of various components of the electric and magnetic fields induced by EM waves are related by Maxwell's equations leads to the relationships specified by (11). It is Li's [6] discovery and her clever exploitation of such relationships that enable application of the ESPRIT algorithm without the need to explicitly employ "doublets" (see [13] for the necessity of using doublets on application of ESPRIT with scalar sensors).

2) To obtain a unique estimate of $\boldsymbol{\Lambda}_{1}^{(p)}$ based on the first equation of (13), it is required that the matrix $\mathbf{E}_{6}^{(p)}$ be of full column rank. Similarly, to determine $\boldsymbol{\Lambda}_{k}^{(p)}$ uniquely, based on the $k$ th equation, where $k=2, \cdots, 9$, it is required that the column rank of $\left[\mathbf{E}_{3}^{(p)}+c_{k} \mathbf{E}_{6}^{(p)}\right]$ be full. In this 
connection, the following assumption ensures the matrices $\Lambda_{k}^{(p)}$ and $\left[\mathbf{E}_{3}^{(p)}+c_{k} \mathbf{E}_{6}^{(p)}\right]$ are of full column rank (see Appendix A for the justifications):

Assumption 4: i) The matrix $\left[\mathbf{e}\left(\phi_{1}, \psi_{1}\right), \cdots, \mathbf{e}\left(\phi_{n}, \psi_{n}\right)\right]$, where $\mathbf{e}$ is as defined in (2), is of full column rank, and $\psi_{k} \neq \pm \pi / 2$ for $k=1, \cdots, n$; ii) $\left(\alpha_{k}, \beta_{k}\right) \neq( \pm \pi / 4,0)$ for $k=1, \cdots, n$.

The assumption imposes some constraints on the number of sensors, the sensor configuration, and the DOA's of the signals. A trivial constraint is that the number of signals must not be more than the number of sensors. Interested readers may refer to [8] and [14] for analyses of the effect of sensor number/configuration and DOA's on the validity of Assumption 4.

3) Although all the necessary DOA and polarization information are available in $\mathbf{E}_{l}^{(p)}$ for $l=1, \cdots, 6$, they are distributed over various $\mathbf{E}_{l}^{(p)}$ in a somewhat complex way and, therefore, cannot be easily extracted. However, a closedform relationship among the diagonal elements of $\mathbf{D}_{k}^{(p)}$ and the DOA and polarization parameters are available, and this allows determination of DOA's and polarizations via an elegant and systematic procedure proposed in [6].

\section{AN INVESTIGATION ON APPLICABILITY OF Li's Method to Partially Polarized Signals}

It is not straightforward to devise a strategy for applying Li's method [6] to PP signals basically because the method is designed for CP signals. Nevertheless, we attempt this by first examining whether the set of relationships among the various measurements of electric and magnetic fields as specified by (11) are still valid for PP signals being model by (7). In this connection, one can show that the same set of relationships holds using the same strategy as that adopted in [6]

$$
\left.\begin{array}{rlrl}
\mathbf{A}_{3} & =\mathbf{A}_{6} \mathbf{D}_{1}^{(u)} & & \\
\mathbf{A}_{1}+b_{k} \mathbf{A}_{4} & =\left(\mathbf{A}_{3}+c_{k} \mathbf{A}_{6}\right) \mathbf{D}_{k}^{(u)}, & & \text { for } k=2, \cdots, 5 \\
\mathbf{A}_{2}+b_{k} \mathbf{A}_{5} & =\left(\mathbf{A}_{3}+c_{k} \mathbf{A}_{6}\right) \mathbf{D}_{k}^{(u)}, & & \text { for } k=6, \cdots, 9
\end{array}\right\}
$$

where $\mathbf{A}_{l}$ is the $(m \times 2 n)$ submatrix of $\mathbf{A}$ comprising the $l$ th, $(6+l)$ th, $\cdots,[6(m-1)+l]$ th rows of $\mathbf{A}$ for $l=1, \cdots, 6$, and $\mathbf{D}_{k}^{(u)}$ is a $(2 n \times 2 n)$ diagonal matrix whose diagonal elements contain DOA information. Moreover, it can be shown using the techniques proposed in [15] that

$$
\mathbf{E}=\mathbf{A T}
$$

where $\mathbf{T}$ is a $(2 n \times 2 n)$ nonsingular matrix, and $\mathbf{E}$ comprises the eigenvectors associated with the $2 n$ largest eigenvalues of R. Consequently, (15) leads to

$$
\left.\begin{array}{rlrl}
\mathbf{E}_{3} & =\mathbf{E}_{6} \boldsymbol{\Lambda}_{1}^{(u)} & \\
\mathbf{E}_{1}+b_{k} \mathbf{E}_{4} & =\left(\mathbf{E}_{3}+c_{k} \mathbf{E}_{6}\right) \boldsymbol{\Lambda}_{k}^{(u)}, & & \text { for } k=2, \cdots, 5 \\
\mathbf{E}_{2}+b_{k} \mathbf{E}_{5} & =\left(\mathbf{E}_{3}+c_{k} \mathbf{E}_{6}\right) \Lambda_{k}^{(u)}, & & \text { for } k=6, \cdots, 9
\end{array}\right\}
$$

where $\boldsymbol{\Lambda}_{k}^{(u)}=\mathbf{T}^{-1} \mathbf{D}_{k}^{(u)} \mathbf{T}$, and $\mathbf{E}_{l}$ is the $(m \times 2 n)$ matrix that is extracted from $\mathbf{E}$ in the same way as $\mathbf{A}_{l}$ is extracted from $\mathbf{A}$ for $l=1, \cdots, 6$. It, therefore, appears that with an estimation procedure similar to that discussed in Section III, i.e., computing $\mathbf{E}_{1}, \cdots, \mathbf{E}_{6}$, determining $\boldsymbol{\Lambda}_{k}^{(u)}$ using (17), and then finding the eigenvalues of $\Lambda_{k}^{(u)}$, one would be able to obtain the DOA information hidden in $\mathrm{D}_{k}^{(u)}$, s. However, a careful analysis shows that the values of $\Lambda_{k}^{(u)}$ cannot be determined uniquely from (17). To demonstrate this, we first establish a relevant lemma, of which the proof is quite straightforward and, hence, not included here.

Lemma 1: The columns of the matrix $\left(\mu \mathbf{A}_{k}+\nu \mathbf{A}_{l}\right)$, where $\mu, \nu \in \mathbb{C}$ and $k, l \in\{1, \cdots, 6\}$, are linearly dependent.

Remark: Note that for the case of $\mathrm{CP}$ signals, one can ensure that the matrices $\left[\mu \mathbf{A}_{k}^{(p)}+\nu \mathbf{A}_{l}^{(p)}\right]$, where $k, l \in$ $\{1, \cdots, 6\}$, are of full column rank via an appropriate constraint on the DOA's of the signals, the sensor configuration, and the use of a sufficient number of sensors. However, Lemma 1 states that for the case of PP signals, the matrices $\left(\mu \mathbf{A}_{k}+\nu \mathbf{A}_{l}\right)$, where $k, l \in\{1, \cdots, 6\}$, are always rank deficient, regardless of the DOA's of the signals, the number of sensors, and sensor configuration.

It follows from Lemma 1 and (16) that $\mathbf{E}_{6}$ as well as $\left(\mathbf{E}_{3}+\right.$ $\left.c_{k} \mathbf{E}_{6}\right)$, for $k=2, \cdots, 9$, are rank deficient. Consequently, there exist infinitely many sets of $\boldsymbol{\Delta}_{k}^{(u)}$, s (with $\mathbf{T}^{-1} \mathbf{D}_{k}^{(u)} \mathbf{T}$ being one candidate) that satisfy

$$
\left.\begin{array}{rlrl}
\mathbf{E}_{3} & =\mathbf{E}_{6} \boldsymbol{\Delta}_{1}^{(u)} & \\
\mathbf{E}_{1}+b_{k} \mathbf{E}_{4} & =\left(\mathbf{E}_{3}+c_{k} \mathbf{E}_{6}\right) \boldsymbol{\Delta}_{k}^{(u)}, & & \text { for } k=2, \cdots, 5 \\
\mathbf{E}_{2}+b_{k} \mathbf{E}_{5} & =\left(\mathbf{E}_{3}+c_{k} \mathbf{E}_{6}\right) \boldsymbol{\Delta}_{k}^{(u)}, & & \text { for } k=6, \cdots, 9 .
\end{array}\right\} \text {. }
$$

Therefore, it is impossible to determine uniquely the DOA's.

Although it turns out that the method proposed in [6] cannot be directly used for PP signals, the analysis carried out here is useful for the development of a new method to be discussed in the next section.

\section{Our New Method for Partially Polarized Signals}

First, note that our method requires that all VS's have the same orientations. Moreover, it requires the following assumption in addition to Assumptions 1 to 3 and Condition i) of Assumption 4.

Assumption 5: For $k=1, \cdots, n, \phi_{k} \neq i \pi / 2$, where $i=-1,0,1,2$, and $\psi_{k} \neq 0$.

Remark: The physical implication of Assumption 5 is that the DOA's of the signals should not lie in the $x-y, x-z$, and $y-z$ planes. Hence, it appears that our method cannot be used for scenarios where signals are known to be confined to the ground plane (i.e., the $x-y$ plane in our context), with which many studies reported in the literature are concerned. However, by reorientating the VS's such that none of the three components of the electric field measurable with them lies in the ground plane, our method becomes applicable to such scenarios.

\section{A. Estimation of Elevations}

As discussed in Section IV, the fact that $\mathbf{E}_{6}$ and $\left(\mathbf{E}_{3}+\right.$ $c_{k} \mathbf{E}_{6}$ ), for $k=2, \cdots, 9$, are rank deficient prohibits unique 
determination of $\boldsymbol{\Delta}_{k}^{(u)}$, s from (18) and, thus, direct application of Li's method [6]. This then motivates the derivation of other relationships among measurements of electric and magnetic fields that are different from those of (18). Indeed, it can be verified using (8) and (9) that

$$
\left(\begin{array}{l}
\mathbf{A}_{1} \\
\mathbf{A}_{2}
\end{array}\right)=\left(\begin{array}{r}
\mathbf{A}_{5} \\
-\mathbf{A}_{4}
\end{array}\right) \mathrm{D}^{(1)}
$$

where

$$
\begin{aligned}
\mathbf{D}^{(1)} & =\operatorname{diag}\left\{d_{1}^{(1)}, \cdots, d_{2 n}^{(1)}\right\} \\
d_{2 i-1}^{(1)} & =\frac{1}{\sin \psi_{i}} \\
d_{2 i}^{(1)} & =\sin \psi_{i}, \quad \text { for } i=1, \cdots, n .
\end{aligned}
$$

Then, it follows from (16) and (19) that

$$
\left(\begin{array}{l}
\mathbf{E}_{1} \\
\mathbf{E}_{2}
\end{array}\right)=\left(\begin{array}{r}
\mathbf{E}_{\tilde{5}} \\
-\mathbf{E}_{4}
\end{array}\right) \mathbf{T}^{-1} \mathbf{D}^{(1)} \mathbf{T}
$$

We now state a crucial theorem.

Theorem 1: Under Condition i) of Assumption 4 and Assumption 5, the matrix $\left[\mathbf{A}_{k}^{T}, \mathbf{A}_{l}^{T}\right]^{T}$, where $k \neq l$, is of full column rank.

Proof: See Appendix B.

Then, by Theorem 1 and (16), the columns of $\left[\mathbf{E}_{\tilde{\partial}}^{T},-\mathbf{E}_{4}^{T}\right]^{T}$ are linearly independent. Thus, the matrix $\boldsymbol{\Delta}^{(1)}$ satisfying

$$
\left(\begin{array}{l}
\mathbf{E}_{1} \\
\mathbf{E}_{2}
\end{array}\right)=\left(\begin{array}{r}
\mathbf{E}_{\tilde{5}} \\
-\mathbf{E}_{4}
\end{array}\right) \boldsymbol{\Delta}^{(1)}
$$

is unique and, hence, must be $\mathbf{T}^{-1} \mathbf{D}^{(1)} \mathbf{T}$. This implies that the eigenvalues of $\boldsymbol{\Delta}^{(1)}$ constitute the diagonal elements $d_{i}^{(1)}$, s of $\mathrm{D}^{(1)}$ for $i=1, \cdots, 2 n$. Exploiting (20) and the fact that $\left|\sin \psi_{i}\right| \leq 1$ and $\left|1 / \sin \psi_{i}\right| \geq 1$, one can take the $n$ eigenvalues with absolute values not greater than 1 to be $d_{2 i}^{(1)}$, s for $i=1, \cdots, n$. Then, the elevations $\psi_{i}$ 's are given by

$$
\psi_{i}=\sin ^{-1}\left[d_{2 i}^{(1)}\right], \quad \text { for } i=1, \cdots, n .
$$

\section{B. Estimation of Azimuths}

Although (19) contains sufficient information for determining the elevations, there is no azimuth information since the matrix $\mathbf{D}^{(1)}$ does not depend on azimuths. We have managed to identify one more equation of the desired form, with a matrix $\mathbf{D}^{(2)}$ containing azimuth information

$$
\left(\begin{array}{l}
\mathbf{A}_{1} \\
\mathbf{A}_{5}
\end{array}\right)=\left(\begin{array}{r}
-\mathbf{A}_{2} \\
\mathbf{A}_{4}
\end{array}\right) \mathbf{D}^{(2)}
$$

where

$$
\begin{aligned}
\mathbf{D}^{(2)} & =\operatorname{diag}\left\{d_{1}^{(2)}, \cdots, d_{2 n}^{(2)}\right\} \\
d_{2 i-1}^{(2)} & =\tan \phi_{i} \\
d_{2 i}^{(2)} & =-\frac{1}{\tan \phi_{i}}, \quad \text { for } i=1, \cdots, n .
\end{aligned}
$$

Then, it follows from (16) and (23) that

$$
\left(\begin{array}{l}
\mathbf{E}_{1} \\
\mathbf{E}_{5}
\end{array}\right)=\left(\begin{array}{r}
-\mathbf{E}_{2} \\
\mathbf{E}_{4}
\end{array}\right) \mathbf{T}^{-1} \mathbf{D}^{(2)} \mathbf{T}
$$

Since the columns of $\left[-\mathbf{E}_{2}^{T}, \mathbf{E}_{4}^{T}\right]^{T}$ are linearly independent [by Theorem 1 and (16)], the matrix $\boldsymbol{\Delta}^{(2)}$ satisfying

$$
\left(\begin{array}{l}
\mathbf{E}_{1} \\
\mathbf{E}_{\tilde{5}}
\end{array}\right)=\left(\begin{array}{r}
-\mathbf{E}_{2} \\
\mathbf{E}_{4}
\end{array}\right) \boldsymbol{\Delta}^{(2)}
$$

must be $\mathbf{T}^{-1} \mathbf{D}^{(2)} \mathbf{T}$. This implies that $d_{i}^{(2)}$ for $i=1, \cdots, 2 n$, which are the eigenvalues of the matrix $\Delta^{(2)}$, constitute the diagonal elements of $\mathrm{D}^{(2)}$.

It follows from (24) that $n$ of the eigenvalues of $\boldsymbol{\Delta}^{(2)}$ correspond to $\tan \phi_{i}$ and the other $n$ of them $-1 / \tan \phi_{i}$. Unlike the case of elevation estimation, there would be ambiguity in estimating the azimuths. Indeed, one would not be able to tell whether a given eigenvalue of $\boldsymbol{\Delta}^{(2)}$ corresponds to $\tan \phi_{i}$ or $-1 / \tan \phi_{i}$ since the allowable values for $\tan \phi_{i}$ and $-1 / \tan \phi_{i}$ coincide exactly. Moreover, each $\tan ^{-1} d_{i}^{(2)}$ yields two possible azimuth estimates within $(-\pi, \pi]$. In Sections V-C and V-D, we shall address these ambiguity issues.

\section{Pairing of Elevation and Azimuth Estimates}

Since the elevation and azimuth estimates are obtained separately using the procedures discussed in Sections V-A and V-B, they have to be appropriately combined. This can be done by determining the pairings of the eigenvalues of $\boldsymbol{\Delta}^{(1)}$ and $\boldsymbol{\Delta}^{(2)}$ (i.e., the correspondence between each one of $d_{1}^{(1)}, \cdots, d_{2 n}^{(1)}$ and $\left.d_{1}^{(2)}, \cdots, d_{2 n}^{(2)}\right)$. For this purpose, the procedure suggested by $\mathrm{Li}$ [6] is suitable. Indeed, we first note that

$$
\left[\boldsymbol{\Delta}^{(1)}\right]^{-1} \boldsymbol{\Delta}^{(2)}=\mathbf{T}^{-1}\left[\mathbf{D}^{(1)}\right]^{-1} \mathbf{D}^{(2)} \mathbf{T}
$$

where $\boldsymbol{\Delta}^{(1)}$ and $\boldsymbol{\Delta}^{(2)}$ are as they appeared in (22) and (26), respectively. First, denote the eigenvalues of $\left[\boldsymbol{\Delta}^{(1)}\right]^{-1} \boldsymbol{\Delta}^{(2)}$ by $d_{i}^{(3)}$ for $i=1, \cdots, 2 n$. Then, it can be deduced that $d_{i}^{(3)}$ is equal to $d_{i}^{(2)} / d_{i}^{(1)}$, where $k \in\{1, \cdots, 2 n\}$, which is the ratio of the $i$ th eigenvalue of $\boldsymbol{\Delta}^{(2)}$ to that of $\boldsymbol{\Delta}^{(1)}$. Moreover, for a $d_{k}^{(3)}$ that corresponds to $d_{i}^{(2)} / d_{i}^{(1)}$, the value $d_{k}^{(3)} d_{i}^{(1)} / d_{i}^{(2)}$ must be equal to 1 . Therefore, we propose first to compute the values $g_{k, l}=\left|1-d_{k}^{(3)} d_{i}^{(1)} / d_{l}^{(2)}\right|$ for $k, l=1, \cdots, 2 n$, and then pairing $d_{i}^{(1)}$ with the $d_{l}^{(2)}$, which gives rise to the minimum among $g_{k, l}$, for $k, l=1, \cdots, 2 n$.

Now, consider the pairs $\left[d_{2 i}^{(1)}, d_{2 i}^{(2)}\right]$ for $i=1, \cdots, n$. Recall that each $d_{2 i}^{(1)}$ yields an elevation estimate $\psi_{i}$ (uniquely), and the azimuth $\phi_{i}$ associated with it is $\tan ^{-1}\left[-1 / d_{2 i}^{(2)}\right]$. Since $\tan ^{-1}\left[-1 / d_{2 i}^{(2)}\right]$ gives rise to two possible values, namely, $\phi_{i}$ or $\phi_{i}+\pi$, we obtain $S_{1}$, which is a set of $n$ possible groups of DOA estimates

$S_{1}=\left\{\left(\phi_{1}, \psi_{1}\right),\left(\phi_{1}+\pi, \psi_{1}\right), \cdots,\left(\phi_{n}, \psi_{n}\right),\left(\phi_{n}+\pi, \psi_{n}\right)\right\}$

where $\left(\phi_{i}, \psi_{i}\right)$ and $\left(\phi_{i}+\pi, \psi_{i}\right)$ form a group, and one of them corresponds to the DOA of an incoming signal. Note that geometrically, $\left(\phi_{i}+\pi, \psi_{i}\right)$ corresponds to the reflection of $\left(\phi_{i}, \psi_{i}\right)$ about the $z$ axis. 


\section{Two Approaches to Tackling Ambiguity in DOA Estimates}

We propose the following two approaches for determining which of the DOA's in $S_{1}$ are the true ones. In particular, we are concerned with whether $\left(\phi_{i}, \psi_{i}\right)$ or $\left(\phi_{i}+\pi, \psi_{i}\right)$ is the true DOA estimate. Note that $\left(\phi_{i}, \psi_{i}\right)$, but not $\left(\phi_{i}+\pi, \psi_{i}\right)$, is the DOA of the $i$ th signal according to our formulation and notations, and thus, it corresponds to the true DOA. However, based on their computed numerical values, we will not know which one corresponds to $\left(\phi_{i}, \psi_{i}\right)$ and which one $\left(\phi_{i}+\pi, \psi_{i}\right)$, besides the fact that they are reflections of each other about the $z$ axis.

1) First Approach/Variant: Let $\mathbf{E}_{N}$ be the $6 m \times(6 m-2 n)$ matrix whose columns are the eigenvectors associated with the $(6 m-2 n)$ smallest eigenvalues of the array covariance matrix R. [The $(6 m-2 n)$ smallest eigenvalues are identical in theory since the signal subspace for $n$ PP signals is $2 n$ instead of $n$ ]. Then, it can be shown that

$$
\left[\mathbf{e}\left(\phi_{i}, \psi_{i}\right) \otimes \mathbf{B}\left(\phi_{i}, \psi_{i}\right)\right]^{H} \mathbf{E}_{N}=\mathbf{0}
$$

for $i=1, \cdots, n$ (refer to [15] for justifications). Thus, for each group of DOA estimates $\left\{\left(\phi_{i}, \psi_{i}\right),\left(\phi_{i}+\pi, \psi_{i}\right)\right\}$, we may determine which one is the true DOA by first constructing the matrices $\mathbf{e}\left(\phi_{i}, \psi_{i}\right) \otimes \mathbf{B}\left(\phi_{i}, \psi_{i}\right)$ and $\mathbf{e}\left(\phi_{i}+\pi, \psi_{i}\right) \otimes \mathbf{B}\left(\phi_{i}+\right.$ $\left.\pi, \psi_{i}\right)$. Then, take $\left(\phi_{i}, \psi_{i}\right)$ to be the true DOA if

$$
\begin{aligned}
& \left\|\left[\mathbf{e}\left(\phi_{i}, \psi_{i}\right) \otimes \mathbf{B}\left(\phi_{i}, \psi_{i}\right)\right]^{H} \mathbf{E}_{N}\right\|_{2} \\
& \quad \leq\left\|\left[\mathbf{e}\left(\phi_{i}+\pi, \psi_{i}\right) \otimes \mathbf{B}\left(\phi_{i}+\pi, \psi_{i}\right)\right]^{H} \mathbf{E}_{N}\right\|_{2}
\end{aligned}
$$

or $\left(\phi_{i}+\pi, \psi_{i}\right)$ otherwise, where $\|\cdot\|_{2}$ denotes the Frobenius norm.

Remarks:

1) Although we have employed the concept of MUSIC (i.e., the steering vector associated with a signal is orthogonal to the noise subspace) to remove ambiguous estimates, our method does not involve a search across the entire array manifold as MUSIC requires. This is a crucial consequence of carrying out the procedures mentioned in Sections V-A through $\mathrm{V}-\mathrm{C}$, which greatly reduces the allowable values of the DOA's to those specified by $S_{1}$.

2) Construction of $\mathbf{e}(\phi, \psi) \otimes \mathbf{B}(\phi, \psi)$ 's requires a priori knowledge about the sensor array such as the sensor positions, and thus, the first variant cannot be used in situations where such knowledge is unavailable.

2) Second Approach/Variant: To avoid construction of $\mathbf{e}\left(\phi_{i}, \psi_{i}\right) \otimes \mathbf{B}\left(\phi_{i}, \psi_{i}\right)$, which requires a priori knowledge about the array, we adopt a strategy different from that of the first variant. This is motivated by the fact that in some applications, such a priori knowledge may not be available. For example, it may be difficult to determine the sensor positions with desired accuracy in some scenarios due to terrain and time constraints. However, this approach would require higher computational cost and would encounter some uniqueness problems as compared with the first approach. These issues will be elaborated upon in Section V-E.

Note that in obtaining the azimuth and elevation estimates in Sections V-A and V-B, we did not make use of the matrices $\mathbf{E}_{3}$ and $\mathbf{E}_{6}$ that contain some additional information about the DOA's, which were captured through the $z$-axis electric sensors and the $z$-axis magnetic sensors, respectively. Such information will be exploited here to tackle the ambiguity problem. First, we recall one of the two possible DOA estimates in the group $\left(\phi_{i}, \psi_{i}\right),\left(\phi_{i}+\pi, \psi_{i}\right)$ of $S_{1}$, where $i=1, \cdots, n$ corresponds to the DOA of an incoming signal, and $\left(\phi_{i}, \psi_{i}\right)$ is the reflection of $\left(\phi_{i}+\pi, \psi_{i}\right)$ about the $z$ axis. For the settings we adopted (see the relevant discussions presented in Section II), the $z$ axis is parallel to one of the three components of electric field measurable with the VS's. Physically, the orientations of the three components with respect to each VS can be unambiguously determined. If we now adopt another coordinate system such that the $z$ axis coincides with one of the other two components, we can obtain another set of DOA estimates (with the use of the procedure proposed) of which the elevations and azimuths are defined with respect to that component. Similarly, we can obtain yet another set of DOA estimates by choosing the $z$ axis to be parallel to the third component of the electric field and then carrying out a similar estimation procedure. Then, the false DOA's in $S_{1}$ may be eliminated by exploiting the fact that the true ones must consistently exist in all three sets of DOA's.

With the above observations in mind, we propose first carrying out a reparamaterization of the DOA and then applying the estimation procedures discussed in Sections V-A through V-C. Indeed, we reparameterize the DOA $(\phi, \psi)$ by $(\hat{\phi}, \hat{\psi})$, as shown in Fig. 2, where $\hat{\phi} \in(-\pi, \pi]$, and $\hat{\psi} \in[-\pi / 2, \pi / 2]$. Note that the parameters $\hat{\phi}$ and $\hat{\psi}$ are the "azimuth" and "elevation" angles with respect to the new coordinate system with axes $\hat{y}, \hat{z}$, and $\hat{x}$, which coincide with the $x, y$, and $z$ axes, respectively, of the previous coordinate system. Without loss of generality, let $\left(\hat{\phi}_{i}, \hat{\psi}_{i}\right)$ denote the same DOA as $\left(\phi_{i}, \psi_{i}\right)$ for $i=1, \cdots, n$. [The actual values of $\left(\hat{\phi}_{i}, \hat{\psi}_{i}\right)$ may be different from those of $\left(\phi_{i}, \psi_{i}\right)$ although they correspond to the same DOA physically since they are defined with respect to different coordinate systems.] Then, we show in Appendix C that (8) and (16) lead to the two equations

$$
\left(\begin{array}{l}
\mathbf{E}_{3} \\
\mathbf{E}_{1}
\end{array}\right)=\left(\begin{array}{r}
\mathbf{E}_{4} \\
-\mathbf{E}_{6}
\end{array}\right)(\hat{\mathbf{T} T})^{-1} \hat{\mathbf{D}}^{(1)}(\hat{\mathbf{T}} \mathbf{T})
$$

where

$$
\begin{aligned}
\hat{\mathbf{D}}^{(1)} & =\operatorname{diag}\left\{\hat{d}_{1}^{(1)}, \cdots, \hat{d}_{2 n}^{(1)}\right\} \\
\hat{d}_{2 i-1}^{(1)} & =\frac{1}{\sin \hat{\psi}_{i}} \\
\hat{d}_{2 i}^{(1)} & =\sin \hat{\psi}_{i}, \quad \text { for } i=1, \cdots, n
\end{aligned}
$$

and $\hat{\mathbf{T}}$ is a nonsingular $(2 n \times 2 n)$ matrix, and

$$
\left(\begin{array}{l}
\mathbf{E}_{3} \\
\mathbf{E}_{4}
\end{array}\right)=\left(\begin{array}{r}
-\mathbf{E}_{1} \\
\mathbf{E}_{6}
\end{array}\right)(\hat{\mathbf{T} T})^{-1} \hat{\mathbf{D}}^{(2)}(\hat{\mathbf{T}} \mathbf{T})
$$

where

$$
\begin{aligned}
\hat{\mathrm{D}}^{(2)} & =\operatorname{diag}\left\{\hat{d}_{1}^{(2)}, \cdots, \hat{d}_{2 n}^{(2)}\right\} \\
\hat{d}_{2 i-1}^{(2)} & =\tan \hat{\phi}_{i} \\
\hat{d}_{2 i}^{(2)} & =-\frac{1}{\tan \hat{\phi}_{i}}, \quad \text { for } i=1, \cdots, n .
\end{aligned}
$$

By Theorem 1 and (16), the matrices $\left[\mathbf{E}_{4}^{T},-\mathbf{E}_{6}^{T}\right]^{T}$ and $\left[-\mathbf{E}_{1}^{T}, \mathbf{E}_{6}^{T}\right]^{T}$ are both of full column rank. Thus, we can 


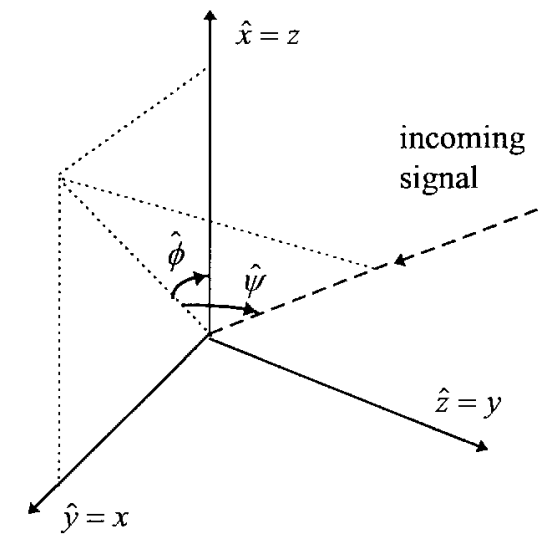

Fig. 2. Reparametrization of the DOA $(\phi, \psi)$ by the parameters $(\hat{\phi}, \hat{\psi})$.

carry out the steps similar to those for estimating $\left(\phi_{i}, \psi_{i}\right)$ 's and obtain another set $S_{2}$ that contains $n$ groups of DOA estimates in terms of $\hat{\phi}$ and $\hat{\psi}$ :

$S_{2}=\left\{\left(\hat{\phi}_{1}, \hat{\psi}_{1}\right),\left(\hat{\phi}_{1}+\pi, \hat{\psi}_{1}\right), \cdots,\left(\hat{\phi}_{n}, \hat{\psi}_{n}\right),\left(\hat{\phi}_{n}+\pi, \hat{\psi}_{n}\right)\right\}$

Geometrically, $\left(\hat{\phi}_{i}+\pi, \hat{\psi}_{i}\right)$ is the reflection of $\left(\hat{\phi}_{i}, \hat{\psi}_{i}\right)$ about the $y$ axis.

Next, we reparameterize the DOA $(\phi, \psi)$ by $(\tilde{\phi}, \tilde{\psi})$, as shown in Fig. 3, and let $\left(\tilde{\phi}_{i}, \tilde{\psi}_{i}\right)$ denote the same DOA as $\left(\phi_{i}, \psi_{i}\right)$ for $i=1, \cdots, n$. Carrying out the steps similar to those presented in Appendix $\mathrm{C}$, we obtain

$$
\left(\begin{array}{l}
\mathbf{E}_{2} \\
\mathbf{E}_{3}
\end{array}\right)=\left(\begin{array}{r}
\mathbf{E}_{6} \\
-\mathbf{E}_{\tilde{3}}
\end{array}\right)(\tilde{\mathbf{T}} \mathbf{T})^{-1} \tilde{\mathbf{D}}^{(1)} \tilde{\mathbf{T}} \mathbf{T}
$$

where

$$
\begin{aligned}
\tilde{\mathbf{D}}^{(1)} & =\operatorname{diag}\left\{\tilde{d}_{1}^{(1)}, \cdots, \tilde{d}_{2 n}^{(1)}\right\} \\
\tilde{d}_{2 i-1}^{(1)} & =\frac{1}{\sin \tilde{\psi}_{i}} \\
\tilde{d}_{2 i}^{(1)} & =\sin \tilde{\psi}_{i}, \quad \text { for } i=1, \cdots, n
\end{aligned}
$$

and $\tilde{\mathbf{T}}$ is a nonsingular $(2 n \times 2 n)$ matrix, and

$$
\left(\begin{array}{l}
\mathbf{E}_{2} \\
\mathbf{E}_{6}
\end{array}\right)=\left(\begin{array}{c}
-\mathbf{E}_{3} \\
\mathbf{E}_{\tilde{3}}
\end{array}\right)(\tilde{\mathbf{T}} \mathbf{T})^{-1} \tilde{\mathbf{D}}^{(2)} \tilde{\mathbf{T}} \mathbf{T}
$$

where

$$
\begin{aligned}
\tilde{\mathbf{D}}^{(2)} & =\operatorname{diag}\left\{\tilde{d}_{1}^{(2)}, \cdots, \tilde{d}_{2 n}^{(2)}\right\} \\
\tilde{d}_{2 i-1}^{(2)} & =\tan \tilde{\phi}_{i} \\
\tilde{d}_{2 i}^{(2)} & =-\frac{1}{\tan \tilde{\phi}_{i}}, \quad \text { for } i=1, \cdots, n .
\end{aligned}
$$

By Theorem 1 and (16), the matrices $\left[\mathbf{E}_{6}^{T},-\mathbf{E}_{5}^{T}\right]^{T}$ and $\left[-\mathbf{E}_{3}^{T}, \mathbf{E}_{5}^{T}\right]^{T}$ are both of full column rank. Thus, with the steps similar to those for estimating $\left(\phi_{i}, \psi_{i}\right)$ 's, we obtain yet another set $S_{3}$, which contains $n$ groups of DOA estimates in terms of $\tilde{\phi}$ and $\tilde{\psi}$ :

$S_{3}=\left\{\left(\tilde{\phi}_{1}, \tilde{\psi}_{1}\right),\left(\tilde{\phi}_{1}+\pi, \tilde{\psi}_{1}\right), \cdots,\left(\tilde{\phi}_{n}, \tilde{\psi}_{n}\right),\left(\tilde{\phi}_{n}+\pi, \tilde{\psi}_{n}\right)\right\}$.

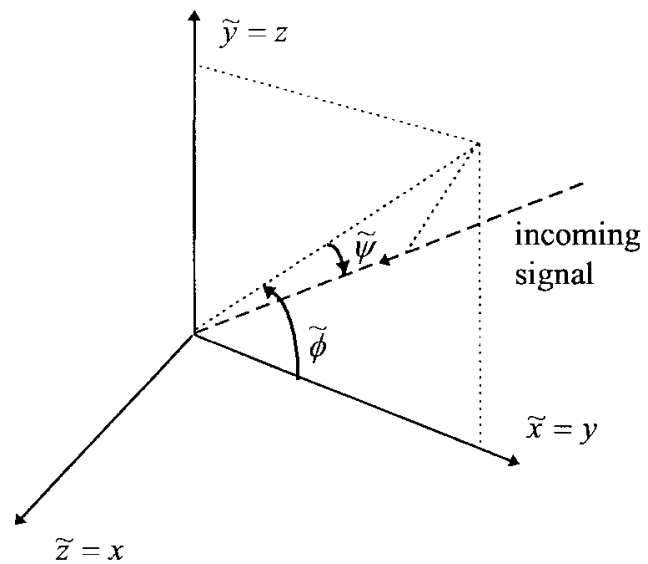

Fig. 3. Reparametrization of the DOA $(\phi, \psi)$ by the parameters $(\tilde{\phi}, \tilde{\psi})$.

Geometrically, $\left(\tilde{\phi}_{i}+\pi, \tilde{\psi}_{i}\right)$ is the reflection of $\left(\tilde{\phi}_{i}, \tilde{\psi}_{i}\right)$ about the $x$ axis.

Now, we shall discuss the strategy for determining which elements in $S_{1}$ correspond to true DOA's with the use of the DOA information available in $S_{2}$ and $S_{3}$. Recall that $S_{1}$ consists of $n$ groups of DOA estimates, namely, $\left\{\left(\phi_{i}, \psi_{i}\right),\left(\phi_{i}+\right.\right.$ $\left.\left.\pi, \psi_{i}\right)\right\}$, for $i=1, \cdots, n$, and for each group, there is one estimate that corresponds to the DOA of an incoming signal. Therefore, it is a challenge to eliminate those that do not correspond to the DOA's of the incoming signals. Since the true DOA's are contained in all three sets $S_{1}, S_{2}$, and $S_{3}$, one sensible approach is to examine whether each DOA in $S_{1}$ matches one in $S_{2}$ and at the same time one in $S_{3}$.

In practice, perfect matching cannot be expected due to errors in estimating each of the elements in $S_{1}, S_{2}$, and $S_{3}$. Therefore, we would need a matching procedure that takes estimation error into considerations. To facilitate our discussions, let us define two unit vectors $\hat{\mathbf{u}}(\hat{\phi}, \hat{\psi})$ and $\tilde{\mathbf{u}}(\tilde{\phi}, \tilde{\psi})$ that point toward $(\hat{\phi}, \hat{\psi})$ and $(\tilde{\phi}, \tilde{\psi})$, respectively

$$
\hat{\mathbf{u}}(\hat{\phi}, \hat{\psi})=\left(\begin{array}{c}
\sin \hat{\phi} \cos \hat{\psi} \\
\sin \hat{\psi} \\
\cos \hat{\phi} \cos \hat{\psi}
\end{array}\right)
$$

and

$$
\tilde{\mathbf{u}}(\tilde{\phi}, \tilde{\psi})=\left(\begin{array}{c}
\sin \tilde{\psi} \\
\cos \tilde{\phi} \cos \tilde{\psi} \\
\sin \tilde{\phi} \cos \tilde{\psi}
\end{array}\right) .
$$

[Recall that $\mathbf{u}(\phi, \psi)$, as defined in (3), is the unit vector pointing toward $(\phi, \psi)$.] Since $\left(\phi_{i}, \psi_{i}\right),\left(\hat{\phi}_{i}, \hat{\psi}_{i}\right)$, and $\left(\tilde{\phi}_{i}, \tilde{\psi}_{i}\right)$ were defined to correspond to the same DOA, $\mathbf{u}\left(\phi_{i}, \psi_{i}\right)$, $\hat{\mathbf{u}}\left(\hat{\phi}_{i}, \hat{\psi}_{i}\right)$, and $\tilde{\mathbf{u}}\left(\tilde{\phi}_{i}, \tilde{\psi}_{i}\right)$ must be the same vector.

Now, we propose first computing the matching indexes $C\left(\phi_{i}, \psi_{i}\right)$ and $C\left(\phi_{i}+\pi, \psi_{i}\right)$ for $i=1, \cdots, n$ (which involve all the DOA's in $S_{1}$ )

$$
\begin{aligned}
C\left(\phi_{i}, \psi_{i}\right)= & \min _{\hat{\mathbf{w}} \in \hat{\Gamma}, \tilde{\mathbf{w}} \in \tilde{\Gamma}}\left\{\left|\cos ^{-1}\left[\mathbf{u}\left(\phi_{i}, \psi_{i}\right) \cdot \hat{\mathbf{w}}\right]\right|\right. \\
& \left.+\left|\cos ^{-1}\left[\mathbf{u}\left(\phi_{i}, \psi_{i}\right) \cdot \tilde{\mathbf{w}}\right]\right|\right\}
\end{aligned}
$$

and

$$
\begin{aligned}
C\left(\phi_{i}+\pi, \psi_{i}\right)= & \min _{\hat{\mathbf{w}} \in \hat{\Gamma}, \tilde{\mathbf{w}} \in \tilde{\Gamma}}\left\{\left|\cos ^{-1}\left[\mathbf{u}\left(\phi_{i}+\pi, \psi_{i}\right) \cdot \hat{\mathbf{w}}\right]\right|\right. \\
& \left.+\left|\cos ^{-1}\left[\mathbf{u}\left(\phi_{i}+\pi, \psi_{i}\right) \cdot \tilde{\mathbf{w}}\right]\right|\right\}
\end{aligned}
$$


where $\hat{\Gamma}$ and $\tilde{\Gamma}$ are defined as

$$
\begin{aligned}
& \hat{\Gamma}=\left\{\hat{\mathbf{u}}\left(\hat{\phi}_{1}, \hat{\psi}_{1}\right), \hat{\mathbf{u}}\left(\hat{\phi}_{1}+\pi, \hat{\psi}_{1}\right), \cdots, \hat{\mathbf{u}}\left(\hat{\phi}_{n}, \hat{\psi}_{n}\right)\right. \\
&\left.\hat{\mathbf{u}}\left(\hat{\phi}_{n}+\pi, \hat{\psi}_{n}\right)\right\} \\
& \tilde{\Gamma}=\left\{\begin{aligned}
\tilde{\mathbf{u}}\left(\tilde{\phi}_{1}, \tilde{\psi}_{1}\right), \tilde{\mathbf{u}}\left(\tilde{\phi}_{1}+\pi, \tilde{\psi}_{1}\right), \cdots, \tilde{\mathbf{u}}\left(\tilde{\phi}_{n}, \tilde{\psi}_{n}\right) \\
\left.\quad \tilde{\mathbf{u}}\left(\tilde{\phi}_{n}+\pi, \tilde{\psi}_{n}\right)\right\}
\end{aligned}\right.
\end{aligned}
$$

and "." is the dot product operator. The term $\mid \cos ^{-1}\left[\mathbf{u}\left(\phi_{i}, \psi_{i}\right)\right.$. w]| yields the angular separation between the unit vectors $\mathbf{u}\left(\phi_{i}, \psi_{i}\right)$ and $\mathbf{w}$, and the value is zero if $\mathbf{u}\left(\phi_{i}, \psi_{i}\right)$ and $\mathbf{w}$ are equal and greater than zero if they are different. Clearly, the matching index $C\left(\phi_{i}, \psi_{i}\right)$ would equal zero in theory, whereas $C\left(\phi_{i}+\pi, \psi_{i}\right)$ yields a relatively large value in general. Therefore, it is sensible to assume that the DOA giving rise to a matching index of relatively lower value is the true DOA.

With the matching procedure discussed above, an element in $S_{1}$ of the form $\left(\phi_{i}+\pi, \psi_{i}\right)$ will be classified as the true DOA if $\mathbf{u}\left(\phi_{i}+\pi, \psi_{i}\right) \in \hat{\Gamma} \cap \tilde{\Gamma}$. The question is whether such an element in fact corresponds to the DOA of an incoming signal. In this connection, the following lemma indicates that in theory, the chance of an element in $S_{1}$ that does not correspond to any true DOA but is erroneously classified as one is very slim (the proof of the lemma is quite straightforward and thus is not included here).

Lemma 2: Given that the DOA sets $\left\{\left(\phi_{1}, \psi_{1}\right), \cdots\right.$, $\left.\left(\phi_{n}, \psi_{n}\right)\right\}, \quad\left\{\left(\hat{\phi}_{1}, \hat{\psi}_{1}\right), \cdots,\left(\hat{\phi}_{n}, \hat{\psi}_{n}\right)\right\} \quad$ and $\left\{\left(\tilde{\phi}_{1}, \tilde{\psi}_{1}\right)\right.$, $\left.\cdots,\left(\tilde{\phi}_{n}, \tilde{\psi}_{n}\right)\right\}$ each contain $n$ pairwise distinct DOA's that are, respectively, defined with respect to the first, second, and third coordinate systems mentioned above, $\left(\phi_{i}, \psi_{i}\right)$, $\left(\hat{\phi}_{i}, \hat{\psi}_{i}\right)$, and $\left(\tilde{\phi}_{i}, \tilde{\psi}_{i}\right)$ correspond to the same DOA for $i=1, \cdots, n$, and $\mathbf{u}, \hat{\Gamma}$, and $\tilde{\Gamma}$ are as defined in (3), (32), and (33), respectively. Then $\mathbf{u}\left(\phi_{i}+\pi, \psi_{i}\right) \in \hat{\Gamma} \cap \tilde{\Gamma}$, where $i \in\{1, \cdots, n\}$, if and only if one of the following conditions holds.

$$
\begin{aligned}
\text { i) } & \psi_{i}= \pm \frac{\pi}{2} ; \\
\text { ii) } & \left(\phi_{i}, \psi_{i}\right)=\left(\phi_{k}+\pi, \psi_{k}\right) ; \\
\text { iii) } & \mathbf{u}\left(\phi_{i}+\pi, \psi_{i}\right)=\hat{\mathbf{u}}\left(\hat{\phi}_{k}, \hat{\psi}_{k}\right)=\tilde{\mathbf{u}}\left(\tilde{\phi}_{l}+\pi, \tilde{\psi}_{l}\right) ; \\
\text { iv) } & \mathbf{u}\left(\phi_{i}+\pi, \psi_{i}\right)=\hat{\mathbf{u}}\left(\hat{\phi}_{k}+\pi, \hat{\psi}_{k}\right)=\tilde{\mathbf{u}}\left(\tilde{\phi}_{l}, \tilde{\psi}_{l}\right) ; \\
\text { v) } & \mathbf{u}\left(\phi_{i}+\pi, \psi_{i}\right)=\hat{\mathbf{u}}\left(\hat{\phi}_{k}+\pi, \hat{\psi}_{k}\right)=\tilde{\mathbf{u}}\left(\tilde{\phi}_{l}+\pi, \tilde{\psi}_{l}\right) ;
\end{aligned}
$$

where $k, l \in\{1, \cdots, n\}, i \neq k, k \neq l$, and $i \neq l$.

\section{Remarks:}

1) Existence of a vector $\mathbf{u}\left(\phi_{i}+\pi, \psi_{i}\right)$ falling in $\hat{\Gamma} \cap \tilde{\Gamma}$ leads to $\left(\phi_{i}+\pi, \psi_{i}\right)$ being accepted as a true DOA. However, this does not necessarily imply incorrect classification. Indeed, Condition i) corresponds to scenarios where there exists an incoming signal with DOA $\left(\phi_{i}, \psi_{i}\right)$ parallel to the $z$ axis. This means that both $\left(\phi_{i}, \psi_{i}\right)$ and $\left(\phi_{i}+\pi, \psi_{i}\right)$ correspond to the DOA of the same signal. On the other hand, Condition ii) corresponds to scenarios where there exist two incoming signals, one of which is the DOA of the reflection of the other about the $z$ axis. Thus, $\left(\phi_{i}, \psi_{i}\right)$ and $\left(\phi_{i}+\pi, \psi_{i}\right)$ each can correspond to the DOA of such an incoming signal.
2) Incorrect classification takes place when Conditions iii) $-v$ ) are satisfied. Conditions iii) $-v$ ) correspond to scenarios where there exist at least three incoming signals. Indeed, Condition iii)/iv) hold when there exists a signal whose DOA coincides with the reflection of the DOA of one other signal about the $z$ axis, and at the same time the reflection of the DOA of another signal about the $x$ axis/ $y$ axis. On the other hand, Condition v) holds when the reflection of the DOA of one signal about the $z$ axis coincides with the reflection of the DOA of one other signal about the $y$ axis and at the same time coincides with the reflection of the DOA of another signal about the $x$ axis.

3) Conditions iii)-v), which are of measure zero in theory, would lead to incorrect classification. In practice, however, measurement noise cannot be avoided, and thus, incorrect classification is of nonzero measure. Indeed, $\left(\phi_{i}+\pi, \psi_{i}\right)$ may be wrongly classified as a true DOA if it is close to any one of those satisfying Conditions iii)-v) in the presence of noise. Nevertheless, Lemma 2 is useful since it indicates that in general, the probability of incorrect classification when near-perfect measurements are available is reasonably low.

The set of DOA's leading to incorrect classification, which has been discussed in Remark 2 of Lemma 2, can be further reduced with additional different parametrization of DOA parameters (or, equivalently, rotation of the coordinate system). Here, we will not attempt to identify the best parametrization scheme as it is a subject by itself. Instead, we shall illustrate the idea by presenting an example. Indeed, we first rotate the original coordinate system by a $\pi / 4$ rotation along the $x-y$ plane followed by a $\pi / 4$ rotation along the new $x-z$ plane (the unit vectors along the $x, y$, and $z$ axes of the new coordinate system are, respectively, $\left(\frac{1}{2}, \frac{1}{2}, 1 / \sqrt{2}\right),(1 / \sqrt{2},-1 / \sqrt{2}, 0)$, and $\left(-\frac{1}{2},-\frac{1}{2}, 1 / \sqrt{2}\right)$ ). Repeating the steps similar to those of obtaining $S_{2}$ in Sections V-A through V-C, one will obtain the fourth set of DOA estimates, say, $S_{4}$. Then, one may identify the true DOA's in $S_{1}$ 's as those that consistently exist in $S_{1}, S_{2}, S_{3}$, and $S_{4}$. With the use of four such different DOA parameterizations, the set of DOA's leading to incorrect classification will be smaller than that obtained by using just the three different DOA parameterizations. In particular, incorrect classification associated with Condition v) of Lemma 2 is alleviated. Indeed, consider a scenario of three incoming signals with DOA's $(-\pi / 4,0),(3 \pi / 4,0)$, and $(-3 \pi / 4,0)$. Then, Condition v) of Lemma 2 is satisfied, and $(\pi / 4,0)$ will be wrongly classified as a true DOA with the use of just $S_{1}, S_{2}$, and $S_{3}$ associated with the first three DOA parameterizations. However, incorrect classification does not occur if we employ $S_{1}, S_{2}, S_{3}$, and $S_{4}$.

\section{E. The Complete DOA Estimation Procedures}

Several key ideas behind our DOA estimation procedures for PP signals and the relevant mathematical justifications have been presented separately in Sections V-A through V-D. It is beneficial to put those ideas together and present the complete estimation procedures, including those essential steps such as 
estimation of the dimension of signal subspace, which we have not addressed.

The First Variant:

Step 1: Compute the estimate of array covariance matrix $\mathbf{R}$

$$
\mathbf{R}_{e}=\frac{1}{N} \sum_{n=1}^{N} \mathbf{y}(t) \mathbf{y}^{H}(t)
$$

where $N$ is the number of snapshots. Then, compute the eigenvalues $\lambda_{1, e} \geq \lambda_{2, e} \geq \cdots \geq$ $\lambda_{6 m, e}$ of $\mathbf{R}_{e}$ and the corresponding eigenvectors $\mathbf{v}_{1, e}, \mathbf{v}_{2, e}, \cdots, \mathbf{v}_{6 m, e}$.

Step 2: Estimate the dimension of signal subspace $2 n_{d}$ using the minimum-description-length-based procedure [16]. (Note that in theory, the number of signals is $n_{d}$.)

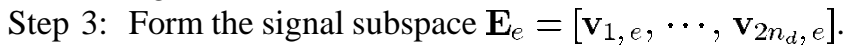
Let $\mathbf{E}_{l, e}$ be the $\left(m \times 2 n_{d}\right)$ submatrix of $\mathbf{E}_{e}$ consisting of the $l$ th, $(6+l)$ th, $\cdots,[6(m-1)+l]$ th rows of $\mathbf{E}_{e}$ for $l=1, \cdots, 6$.

Step 4: Compute, using the total least squares algorithm [13], the matrices $\boldsymbol{\Delta}_{e}^{(1)}$ and $\boldsymbol{\Delta}_{e}^{(2)}$ that satisfy

$$
\begin{aligned}
& \left(\begin{array}{l}
\mathbf{E}_{1, e} \\
\mathbf{E}_{2, e}
\end{array}\right)=\left(\begin{array}{r}
\mathbf{E}_{5, e} \\
-\mathbf{E}_{4, e}
\end{array}\right) \Delta_{e}^{(1)} \\
& \left(\begin{array}{l}
\mathbf{E}_{1, e} \\
\mathbf{E}_{5, e}
\end{array}\right)=\left(\begin{array}{r}
-\mathbf{E}_{2, e} \\
\mathbf{E}_{4, e}
\end{array}\right) \boldsymbol{\Delta}_{e}^{(2)} .
\end{aligned}
$$

Step 5: Compute $d_{1, e}^{(1)}, \cdots, d_{2 n_{d}}^{(1)}, e$, which are the eigenvalues of $\boldsymbol{\Delta}_{e}^{(1)}$, and $d_{1, e}^{(2)}, \cdots, d_{2 n_{d}, e}^{(2)}$ of $\boldsymbol{\Delta}_{e}^{(2)}$.

Step 6: Compute $d_{1, e}^{(3)}, \cdots, d_{2 n_{d}, e}^{(3)}$, which are the eigenvalues of $\left[\boldsymbol{\Delta}_{e}^{(1)}\right]^{-1} \boldsymbol{\Delta}_{e}^{(2)}$, and then for $i=1, \cdots, 2 n_{d}$, compute the values $\left|1-d_{k, e}^{(3)} d_{i, e}^{(1)} / d_{l, e}^{(2)}\right|$ for $k, l=$ $1, \cdots, 2 n_{d}$. Pair $d_{i, e}^{(1)}$ with the $d_{l, e}^{(2)}$ associated with the minimum of these values.

Step 7: Choose the $n_{d}$ smallest eigenvalues of $\boldsymbol{\Delta}_{e}^{(1)}$, and identify them as $d_{2 i, e}^{(1)}$ for $i=1, \cdots, n_{d}$. In addition, identify the eigenvalue of $\Delta_{e}^{(2)}$ associated with $d_{2 i, e}^{(1)}$ (based on the pairing relationship established via Step 6) as $d_{2 i, e}^{(2)}$. Estimate the elevation by $\psi_{i, e}=\operatorname{Re}\left[\sin ^{-1} d_{2 i, e}^{(1)}\right]$ for $i=1, \cdots, n_{d}$ and the corresponding azimuth by $\phi_{i, e}=\operatorname{Re}\left\{\tan ^{-1}\left[-1 / d_{2 i, e}^{(2)}\right]\right\}$, where $\operatorname{Re}(x)$ denotes the real part of $x$. A set of $n_{d}$ groups of DOA estimates can then be obtained

$$
\begin{array}{r}
S_{1, e}=\left\{\left(\phi_{1, e}, \psi_{1}, e\right),\left(\phi_{1, e}+\pi, \psi_{1}, e\right), \cdots,\right. \\
\left.\left(\phi_{n_{d}, e}, \psi_{n_{d}, e}\right),\left(\phi_{n_{d}, e}+\pi, \psi_{n_{d}, e}\right)\right\} .
\end{array}
$$

Step 8: Form the noise subspace $\mathbf{E}_{N, e}=\left[\mathbf{v}_{2 n_{d}+1, e}, \cdots\right.$, $\left.\mathbf{v}_{6 m}, e\right]$. For each group of DOA estimates $\left(\phi_{i, e}, \psi_{i, e}\right)$ and $\left(\phi_{i, e}+\pi, \psi_{i, e}\right)$ in $S_{1, e}$, where $i=1, \cdots, n_{d}$, take $\left(\phi_{i, e}, \psi_{i, e}\right)$ as a true DOA if

$$
\begin{aligned}
& \left\|\left[\mathbf{e}\left(\phi_{i, e}, \psi_{i, e}\right) \otimes \mathbf{B}\left(\phi_{i, e}, \psi_{i, e}\right)\right]^{H} \mathbf{E}_{N, e}\right\|_{2} \\
& \quad \leq \|\left[\mathbf{e}\left(\phi_{i, e}+\pi, \psi_{i, e}\right) \otimes \mathbf{B}\left(\phi_{i, e}+\pi, \psi_{i, e}\right)\right]^{H} \\
& \quad \mathbf{E}_{N, e} \|_{2}
\end{aligned}
$$

or take $\left(\phi_{i, e}+\pi, \psi_{i, e}\right)$ as a true DOA otherwise.
The Second Variant:

Step 1: This step simply consists of Steps $1-7$ of the first variant.

Step 2: Adopt another coordinate system and reparameterize the DOA by $(\hat{\phi}, \hat{\psi})$ in the way described in Section V-D2. Replace (34) with

$$
\left(\begin{array}{l}
\mathbf{E}_{3, e} \\
\mathbf{E}_{1, e}
\end{array}\right)=\left(\begin{array}{r}
\mathbf{E}_{4, e} \\
-\mathbf{E}_{6, e}
\end{array}\right) \hat{\boldsymbol{\Delta}}_{e}^{(1)}
$$

and (35) with

$$
\left(\begin{array}{l}
\mathbf{E}_{3, e} \\
\mathbf{E}_{4, e}
\end{array}\right)=\left(\begin{array}{r}
-\mathbf{E}_{1, e} \\
\mathbf{E}_{6, e}
\end{array}\right) \hat{\boldsymbol{\Delta}}_{e}^{(2)}
$$

and repeat Steps 4-7 of the first variant. Then, another set of $n_{d}$ groups of DOA estimates in terms of $(\hat{\phi}, \hat{\psi})$ will be obtained

$$
\begin{aligned}
S_{2, e}=\{ & \left(\hat{\phi}_{1, e}, \hat{\psi}_{1, e}\right),\left(\hat{\phi}_{1, e}+\pi, \hat{\psi}_{1, e}\right), \cdots \\
& \left.\left(\hat{\phi}_{n_{d}, e}, \hat{\psi}_{n_{d}, e}\right),\left(\hat{\phi}_{n_{d}, e}+\pi, \hat{\psi}_{n_{d}, e}\right)\right\} .
\end{aligned}
$$

Step 3: Adopt yet another coordinate system and reparameterize the DOA by $(\tilde{\phi}, \tilde{\psi})$ in the way described in Section V-D2. Replace (34) with

$$
\left(\begin{array}{l}
\mathbf{E}_{2, e} \\
\mathbf{E}_{3, e}
\end{array}\right)=\left(\begin{array}{r}
\mathbf{E}_{6, e} \\
-\mathbf{E}_{5, e}
\end{array}\right) \tilde{\Delta}_{e}^{(1)}
$$

and (35) with

$$
\left(\begin{array}{l}
\mathbf{E}_{2, e} \\
\mathbf{E}_{6, e}
\end{array}\right)=\left(\begin{array}{c}
-\mathbf{E}_{3, e} \\
\mathbf{E}_{5, e}
\end{array}\right) \tilde{\boldsymbol{\Delta}}_{e}^{(2)}
$$

and repeat Steps 4-7 of the first variant. Then, the third set of $n_{d}$ groups of DOA estimates in terms of $(\tilde{\phi}, \widetilde{\psi})$ will be obtained

$$
\begin{aligned}
S_{3, e}=\{ & \left(\tilde{\phi}_{1, e}, \tilde{\psi}_{1, e}\right),\left(\tilde{\phi}_{1, e}+\pi, \tilde{\psi}_{1}, e\right), \cdots \\
& \left.\left(\tilde{\phi}_{n_{d}, e}, \tilde{\psi}_{n_{d}, e}\right),\left(\tilde{\phi}_{n_{d}, e}+\pi, \tilde{\psi}_{n_{d}, e}\right)\right\} .
\end{aligned}
$$

Step 4: For each group of DOA estimates $\left(\phi_{i, e}, \psi_{i, e}\right)$ and $\left(\phi_{i, e}+\pi, \psi_{i, e}\right)$ in $S_{1, e}$, where $i=1, \cdots, n_{d}$, compute the matching indexes $C\left(\phi_{i, e}, \psi_{i, e}\right)$ and $C\left(\phi_{i, e}+\pi, \psi_{i, e}\right)$

$$
\begin{aligned}
C\left(\phi_{i}, \psi_{i}\right)= & \min _{\hat{\mathbf{w}} \subset \hat{\Gamma}_{e}, \tilde{\mathbf{w}} \subset \tilde{\Gamma}_{e}}\left\{\left|\cos ^{-1}\left[\mathbf{u}\left(\phi_{i}, \psi_{i}\right) \cdot \hat{\mathbf{w}}\right]\right|\right. \\
& \left.+\left|\cos ^{-1}\left[\mathbf{u}\left(\phi_{i}, \psi_{i}\right) \cdot \tilde{\mathbf{w}}\right]\right|\right\} \\
C\left(\phi_{i}+\pi, \psi_{i}\right)= & \min _{\hat{\mathbf{w}} \in \hat{\Gamma}_{e}, \tilde{\mathbf{w}} \subset \tilde{\Gamma}_{e}}\left\{\left(\mid \cos ^{-1}\left[\mathbf{u}\left(\phi_{i}+\pi, \psi_{i}\right)\right.\right.\right. \\
& \left.\cdot \hat{\mathbf{w}}]|+| \cos ^{-1}\left[\mathbf{u}\left(\phi_{i}+\pi, \psi_{i}\right) \cdot \tilde{\mathbf{w}}\right] \mid\right\}
\end{aligned}
$$

where

$$
\begin{array}{r}
\hat{\Gamma}_{e}=\left\{\hat{\mathbf{u}}\left(\hat{\phi}_{1, e}, \hat{\psi}_{1, e}\right), \hat{\mathbf{u}}\left(\hat{\phi}_{1, e}+\pi, \hat{\psi}_{1}, e\right), \cdots\right. \\
\left.\hat{\mathbf{u}}\left(\hat{\phi}_{n_{d}, e}, \hat{\psi}_{n_{d}}, e\right), \hat{\mathbf{u}}\left(\hat{\phi}_{n_{d}}, e+\pi, \hat{\psi}_{n_{d}, e}\right)\right\}
\end{array}
$$

and

$$
\begin{aligned}
\tilde{\Gamma}_{e}=\left\{\tilde{\mathbf{u}}\left(\tilde{\phi}_{1, e}, \tilde{\psi}_{1}, e\right), \tilde{\mathbf{u}}\left(\tilde{\phi}_{1, e}+\pi, \tilde{\psi}_{1}, e\right), \cdots\right. \\
\left.\tilde{\mathbf{u}}\left(\tilde{\phi}_{n_{d}}, e, \tilde{\psi}_{n_{d}}, e\right), \tilde{\mathbf{u}}\left(\tilde{\phi}_{n_{d}}, e+\pi, \tilde{\psi}_{n_{d}, e}\right)\right\} .
\end{aligned}
$$

Take $\left(\phi_{i, e}, \psi_{i, e}\right)$ as a valid DOA estimate if $C\left(\phi_{i, e}, \psi_{i, e}\right) \leq C\left(\phi_{i, e}+\pi, \psi_{i, e}\right)$, or take $\left(\phi_{i, e}+\pi, \psi_{i, e}\right)$ otherwise. 


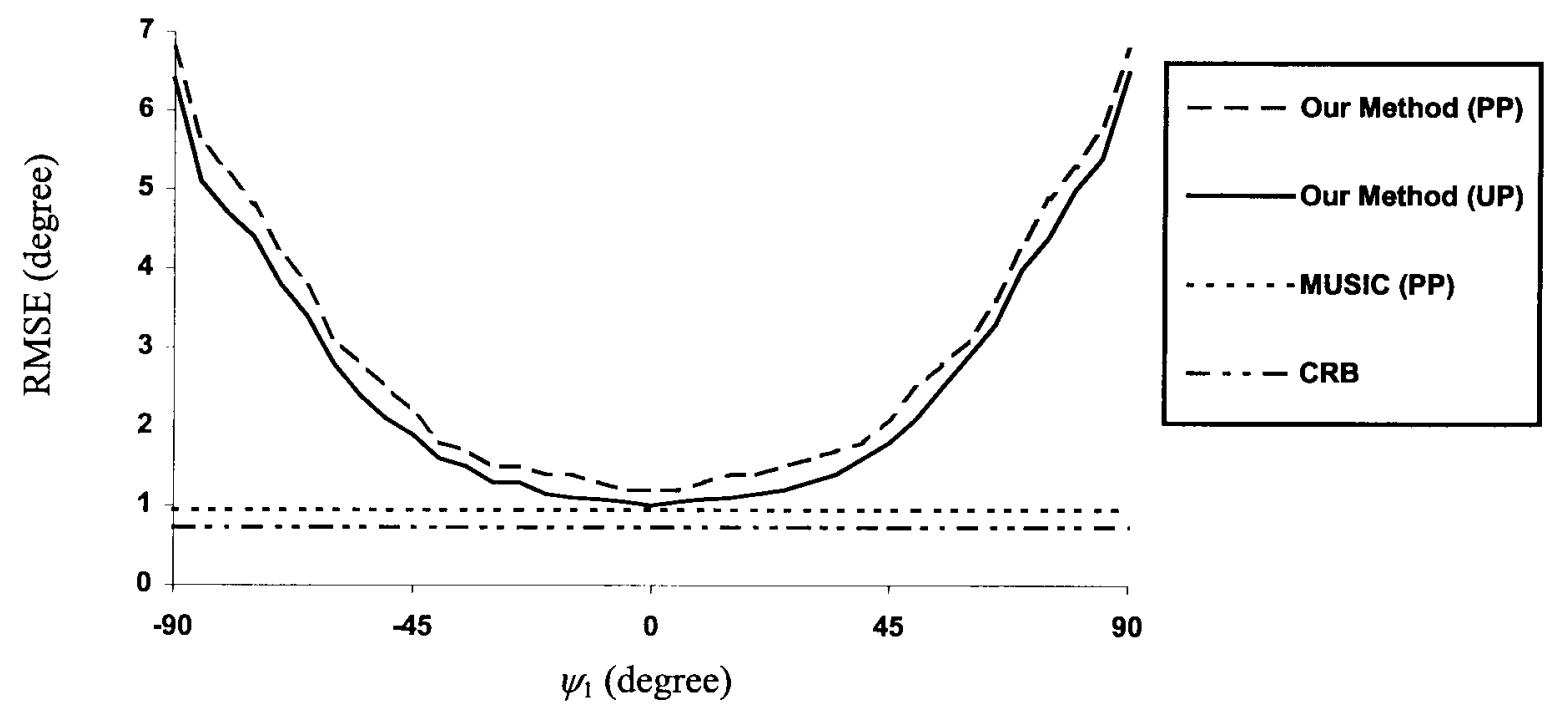

Fig. 4. RMSE of the elevation estimate, and the CRB, of one signal impinging on one vector sensor. The azimuth of the signal is fixed at $45^{\circ}$, and the elevation $\psi_{1}$ varies from $-90^{\circ}$ to $90^{\circ}$. The words "PP" and "UP" in the legend in the figure indicate that the curve corresponds to, respectively, scenario with a PP signal and that with a UP signal. (The CRB's are identical for the two different scenarios, whereas the RMSE of MUSIC for a UP signal coincides with that of the CRB and, thus, is not shown here.)

Remark: The strength of the second variant of our method is that it does not require a priori knowledge about the array configurations, but it will lead to incorrect classification for scenarios where the DOA's of the signals satisfy Conditions iii) $-v)$. On the other hand, the first variant is computationally more efficient than the second variant (the computation cost of Steps $2-4$ of the latter is clearly much higher than that of Step 8 of the former) and does not have the ambiguity problem. However, it requires the information about the array configurations.

\section{Simulation Results}

We shall now present some numerical examples to demonstrate the effectiveness of the method we proposed. Altogether, two experiments were conducted. In each of the experiments, we generated 500 Monte Carlo runs, and for each run, 100 snapshots were generated. The signal-to-noise ratio (SNR) is defined as the ratio of the incident signal power to the noise power received at each VS and is equal to $10 \log _{10}$ $\left(\sigma_{i}^{2}+\hat{\sigma}_{i}^{2}\right) / \sigma_{n}^{2}$, where $\sigma_{i}^{2}$ and $\hat{\sigma}_{i}^{2}$ are, respectively, the powers of the CP and UP components of the $i$ th signal, and $\sigma_{n}^{2}$ is the noise power. The SNR's of each signals were fixed at 15 $\mathrm{dB}$. We compared the performance of our proposed estimator with MUSIC [15] and with the Cramér-Rao bound (CRB) given by [1, Eq. (3.4)]. MUSIC basically searches for the "steering matrices" in the array manifold of UP signals, i.e., $\{\mathbf{e}(\phi, \psi) \otimes \mathbf{B}(\phi, \psi) \mid \phi \in(-\pi, \pi], \psi \in[-\pi / 2, \pi / 2]\}$, that intersect the noise subspace and then computes the DOA's accordingly. A step size of $0.1^{\circ}$ was used to perform the search.

In the first experiment, we simulated one signal impinging on one VS. The azimuth of the signal was fixed at $45^{\circ}$, and the elevation was varied from $-90^{\circ}$ to $90^{\circ}$ in steps of $5^{\circ}$. We consider two scenarios with, respectively, a UP signal and a PP signal with polarization parameters $\alpha_{1}=\beta_{1}=0^{\circ}$ and $\sigma_{1}^{2}=\hat{\sigma}_{1}^{2}=0.5$ (the degree of polarization is 0.5). Fig. 4 shows the root-mean-square errors (RMSE's) of the elevation estimates obtained with our method for the scenarios where there exists a UP signal as well as those for the PP signal. It also shows the estimates obtained with MUSIC for the scenarios where there exists a PP signal and the CRB. (The CRB's for the two scenarios are identical, and the RMSE of MUSIC for a UP signal is very close to the CRB and is, thus, not shown.)

It is shown in Fig. 4 that for elevation ranging from $-60^{\circ}$ to $60^{\circ}$, the RMSE's of our method remained below $3^{\circ}$ and were slightly larger than those of MUSIC. Although the RMSE of our method rose to about $7^{\circ}$ when elevation was $\pm 90^{\circ}$, it will not result in serious setback in some applications such as estimation of the DOA's of skywaves, where signals of high elevation are of less concern. Indeed, skywaves are often exploited for long distance communications, and high elevation skywaves often correspond to relatively nearby transmitting sources.

Although the accuracy of the estimates obtained by our method is poorer than that by MUSIC, our method is computationally more efficient as it does not require searching across the entire array manifold. Moreover, one variant of our method does not require a priori knowledge about the sensor positions. In this connection, it is worth mentioning that the performance of MUSIC depends greatly on the accuracy of the array manifold, which in turn is dependent on the accuracy of the sensor positions. If the sensor positions are not known precisely, the DOA estimates of MUSIC could degrade significantly. In cases where high-accuracy DOA estimates are required and the sensor positions are known to desired accuracy, our method can be used to provide initial estimates. Then, more accurate estimates can be obtained with MUSIC via performing a search across only those regions of the array manifold that are close to the steering matrices associated with the initial estimates. This approach will lead to considerable reduction in computational cost. 


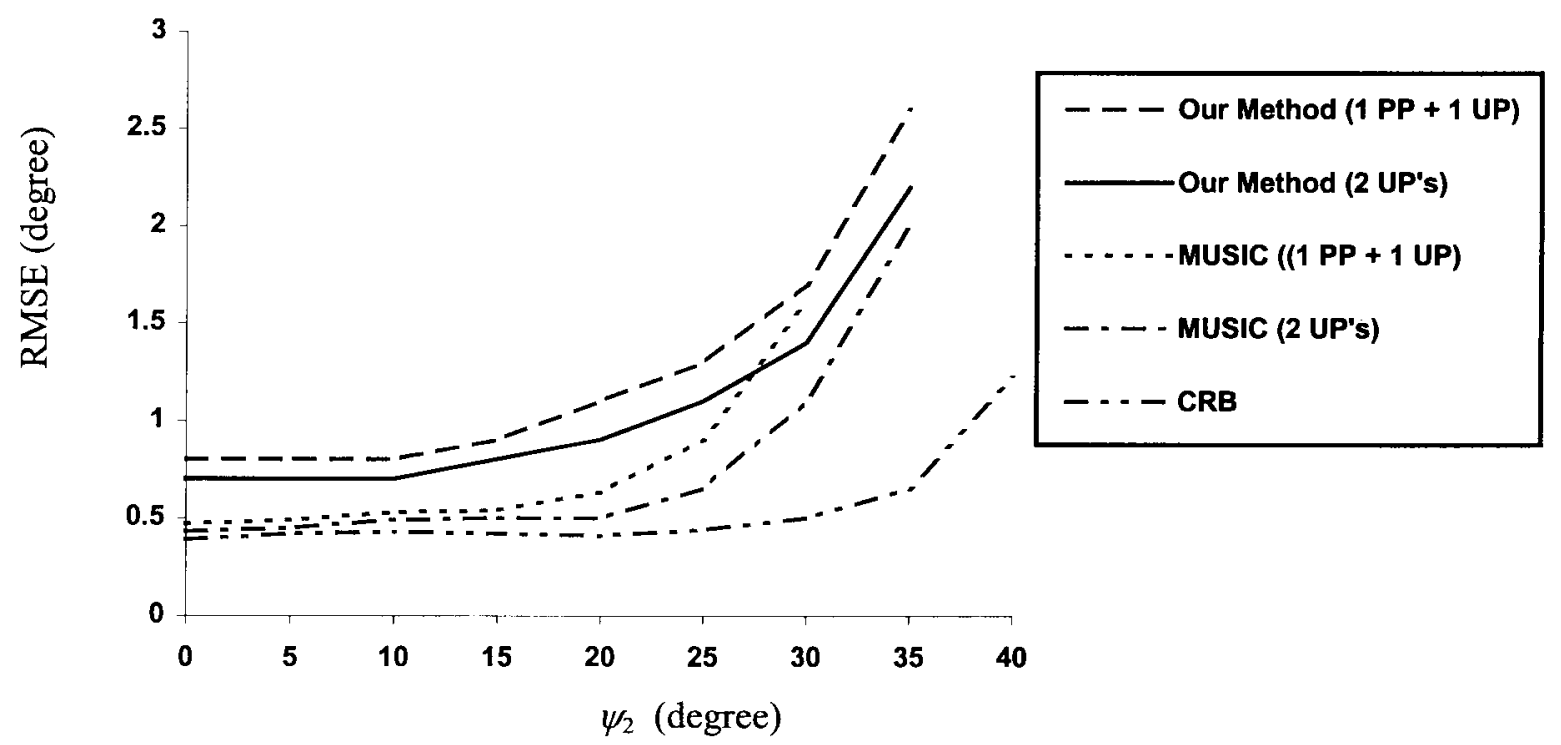

Fig. 5. RMSE of the elevation estimate of the second signal for scenarios of two signals impinging on an array of four vector sensors with coordinates $(0$, $0,0),(1,0,0) \lambda,(0,1,0) \lambda,(1,1,0) \lambda$. The azimuths of the two signals are fixed at $45^{\circ}$, the elevation of the first signal is fixed at $45^{\circ}$, and the second $\left(\psi_{2}\right)$ varies from $0^{\circ}$ to $45^{\circ}$. The words " 1 PP +1 UP" and " 2 UP' $s$ " in the legend in the figure indicate that the curve corresponds to, respectively, the scenario where there exists a UP signal and a PP signal and that where there exist two UP signals. (The CRB's are identical for the two different scenarios.)

Note that from Fig. 4, we can see that the RMSE's of our method, as well as for MUSIC, for the PP signal were larger than those for UP signal. This is due to the fact that our method, and MUSIC by the way we apply it (see the first paragraph of this section), exploits only the UP component of the PP signal for DOA estimation. Further experiments (which are not shown here) indicate that when the degree of polarization is about 0.9 , the DOA estimate of our method becomes quite poor. Note that our method will not be able to estimate the DOA's of a CP signal, and, in contrast, the method proposed by $\mathrm{Li}$ [6] requires all the signals to be $\mathrm{CP}$.

In the second experiment, we simulated two uncorrelated signals impinging on an array of four VS's. The sensor coordinates were $(0,0,0),(1,0,0) \lambda,(0,1,0) \lambda$, and $(1,1,0) \lambda$, where $\lambda$ denotes the wavelength of the signals. We consider two different scenarios: one where both the signals are UP and the other where one of the signals is UP and the other PP. For both scenarios, the DOA of the first signal was $\left(\phi_{1}, \psi_{1}\right)=$ $\left(45^{\circ}, 45^{\circ}\right)$, and the second signal was $\left(\phi_{2}, \psi_{2}\right)=\left(45^{\circ}, \psi_{2}\right)$, where the value of $\psi_{2}$ was varied from 0 to $35^{\circ}$ in steps of $5^{\circ}$. Moreover, for the second scenario, the first signal is UP, and the second signal is PP with parameters $\left(\alpha_{2}, \beta_{2}\right)=\left(90^{\circ}, 0^{\circ}\right)$ and $\sigma_{2}^{2}=\hat{\sigma}_{2}^{2}=0.5$ (the degree of polarization is 0.5 ). Fig. 5 shows the RMSE's of the elevation estimates of the second signal obtained with our method, as well as those with MUSIC, and the CRB for the scenarios where there exist two UP signals and those where there exist one UP signal and one PP signal. (The CRB's for the two scenarios are identical.) It is apparent that the RMSE of our method was only slightly larger than those of MUSIC for the range of elevation from 0 to $30^{\circ}$. For elevation between $0^{\circ}$ to $20^{\circ}$, our method was at most $0.65^{\circ}$ poorer than the CRB.

Note that both variants of our method have led to the same results shown in Figs. 4 and 5. Indeed, the difference between the two variants lies in the approach to determining which of the elements of $S_{1}$ are the true DOA's, and for the experiments conducted, both variants have led to the correct choice of true DOA's.

\section{CONCLUSION}

We first examined the possibility of applying Li's method [6] to partially polarized signals. Indeed, we began by studying whether the relationships among the measurements of electric and magnetic fields obtainable with vector sensors that $\mathrm{Li}$ [6] had established are also valid for partially polarized signals. Although the same relationships hold, we discovered that the information they contain is not sufficient for determining uniquely the DOA's for partially polarized signals. Subsequently, we derived a new set of relationships and an appropriate estimation method with two variants. Our method is computationally efficient, and one of the variants does not require a priori information about the array system such as sensor positions. Moreover, simulation results have demonstrated that our method is effective.

While Li's method [6] works well with only completely polarized signals, our method is applicable to only partially polarized signals. Therefore, it remains a challenge to develop an estimation method that possesses the strengths of both Li's and of our method for scenarios where completely polarized signals and partially polarized signals co-exist.

\section{APPENDIX A}

\section{THE IMPLICATION OF ASSUMPTION 4}

We shall establish that Assumption 4 ensures the matrices $\mathbf{E}_{6}^{(p)}$ and $\left[\mathbf{E}_{3}^{(p)}+c_{k} \mathbf{E}_{6}^{(p)}\right]$ are of full column rank. First, let $\boldsymbol{\Omega} \triangleq\left[\mathbf{e}\left(\phi_{1}, \psi_{1}\right), \cdots, \mathbf{e}\left(\phi_{n}, \psi_{n}\right)\right]$. From (12), we have

$$
\mathbf{E}_{3}^{(p)}+c_{k} \mathbf{E}_{6}^{(p)}=\left[\mathbf{A}_{3}^{(p)}+c_{k} \mathbf{A}_{6}^{(p)}\right] \mathbf{T}^{(p)}=\mathbf{\Omega} \mathbf{F} \mathbf{T}^{(p)}
$$


where $\mathbf{F}=\operatorname{diag}\left\{f_{1}, \cdots, f_{k}\right\}$, and $f_{i}=\left(-\sin \alpha_{i} \cos \beta_{i}\right.$ $\left.+j \cos \alpha_{i} \sin \beta_{i}\right) \cos \psi_{i}+c_{k}\left(\cos \alpha_{i} \cos \beta_{i}+\right.$ $\left.j \sin \alpha_{i} \sin \beta_{i}\right) \cos \psi_{i}$. Under Assumption 4, we have $\psi_{i} \neq \pm 90^{\circ}$ and $\left(\alpha_{i}, \beta_{i}\right) \neq( \pm \pi / 4,0)$ for $i=1, \cdots, n$, implying that $f_{i}$ is nonzero for $i=1, \cdots, n$. Consequently, $\mathbf{F}$ is of full column rank. Moreover, $\boldsymbol{\Omega}$ is of full column rank under Assumption 4, and $\mathbf{T}^{(p)}$, as it is defined [see (12)], is also of full column rank. These facts imply that $\left[\mathbf{E}_{3}^{(p)}+c_{k} \mathbf{E}_{6}^{(p)}\right]$ is of full column rank. Using similar strategy, we can show that $\mathbf{E}_{6}^{(p)}$ is of full column rank.

\section{APPENDIX B \\ PROOF OF THEOREM 1}

We first show that under Condition i) of Assumption 4, the matrix $\left[\mathbf{A}_{k}^{T}, \mathbf{A}_{l}^{T}\right]^{T}$, where $k \neq l$, is of full column rank if

$$
\operatorname{det}\left[\begin{array}{l}
\mathbf{b}_{k}\left(\phi_{i}, \psi_{i}\right) \\
\mathbf{b}_{l}\left(\phi_{i}, \psi_{i}\right)
\end{array}\right] \neq 0, \quad \text { for } i=1, \cdots, n
$$

where $\mathbf{b}_{k}\left(\phi_{i}, \psi_{i}\right) \in \mathbb{C}^{1 \times 2}$ denotes the $k$ th row of the $6 \times 2$ matrix $\mathbf{B}\left(\phi_{i}, \psi_{i}\right)$. Indeed, the matrix $\left[\mathbf{e}\left(\phi_{1}, \psi_{1}\right), \cdots, \mathbf{e}\left(\phi_{n}, \psi_{n}\right)\right]$ is of full column rank under Condition i) of Assumption 4, and this implies that there exists a nonsingular $(m \times m)$ matrix $\mathbf{M}$ such that

$$
\mathbf{M}\left[\mathbf{e}\left(\phi_{1}, \psi_{1}\right), \cdots, \mathbf{e}\left(\phi_{n}, \psi_{n}\right)\right]=\left[\begin{array}{c}
\mathbf{I}_{n} \\
\mathbf{0}_{(m-n) \times n}
\end{array}\right] .
$$

Since $\mathbf{A}_{k}=\left[\mathbf{e}\left(\phi_{1}, \psi_{1}\right) \mathbf{b}_{k}\left(\phi_{1}, \psi_{1}\right), \cdots, \mathbf{e}\left(\phi_{n}, \psi_{n}\right) \mathbf{b}_{k}\left(\phi_{n}\right.\right.$, $\left.\left.\psi_{n}\right)\right]$ for $k=1, \cdots, 6$, we can express it as

$$
\mathbf{A}_{k}=\left[\mathbf{e}\left(\phi_{1}, \psi_{1}\right), \cdots, \mathbf{e}\left(\phi_{n}, \psi_{n}\right)\right] \mathbf{N}_{k}
$$

where we have (38a), shown at the bottom of the page.

It then follows from (37) and (38) that

$$
\left(\begin{array}{cc}
\mathbf{M} & \mathbf{0}_{m \times m} \\
\mathbf{0}_{m \times m} & \mathbf{M}
\end{array}\right)\left(\begin{array}{l}
\mathbf{A}_{k} \\
\mathbf{A}_{l}
\end{array}\right)=\left(\begin{array}{l}
\mathbf{N}_{k} \\
\mathbf{N}_{l}
\end{array}\right) .
$$

Since $\mathbf{M}$ is nonsingular, the ranks of the matrices $\left[\mathbf{A}_{k}^{T}, \mathbf{A}_{l}^{T}\right]^{T}$ and $\left[\mathbf{N}_{k}^{T}, \mathbf{N}_{l}^{T}\right]^{T}$ are the same. Thus, it suffices to show that the matrix $\left[\mathbf{N}_{k}^{T}, \mathbf{N}_{l}^{T}\right]^{T}$ is of full column rank if (36) holds, or equivalently, the null space of $\left[\mathbf{N}_{k}^{T}, \mathbf{N}_{l}^{T}\right]^{T}$ contains only the zero vector if (36) holds. To see this, let $\mathbf{c}=$ $\left[c_{1}, c_{2}, \cdots, c_{2 n}\right]^{T}$ be a vector in $\mathbb{C}^{2 n \times 1}$ such that

$$
\left(\begin{array}{l}
\mathrm{N}_{k} \\
\mathrm{~N}_{l}
\end{array}\right) \mathbf{c}=\mathbf{0}_{2 n \times 1} \text {. }
$$

We shall show that $c_{i}=0$ for $i=1, \cdots, 2 n$. From the $i$ th row and the $(n+i)$ th row of (39), for $i=1, \cdots, n$, we obtain

$$
\left[\begin{array}{l}
\mathbf{b}_{k}\left(\phi_{i}, \psi_{i}\right) \\
\mathbf{b}_{l}\left(\phi_{i}, \psi_{i}\right)
\end{array}\right]\left[\begin{array}{c}
c_{i} \\
c_{n+i}
\end{array}\right]=\left[\begin{array}{l}
0 \\
0
\end{array}\right], \quad \text { for } i=1, \cdots, n .
$$

Clearly, if (36) holds, then $c_{i}$ and $c_{n+i}$ satisfying the above equation must be 0 for $i=1, \cdots, n$, and hence, the matrix $\left[\mathbf{N}_{k}^{T}, \mathbf{N}_{l}^{T}\right]^{T}$ is of full column rank. With this, we establish that under Condition i) of Assumption 4, the matrix $\left[\mathbf{A}_{k}^{T}, \mathbf{A}_{l}^{T}\right]^{T}$ is of full column rank if (36) holds.

Now, we shall show that under Assumption 5, (36) holds. To see this, we assume the contrary that Assumption 5 holds but that (36) is invalid. This implies that there exist $k$ and $l$, where $k \neq l$, such that

$$
\operatorname{det}\left[\begin{array}{l}
\mathbf{b}_{k}\left(\phi_{i}, \psi_{i}\right) \\
\mathbf{b}_{l}\left(\phi_{i}, \psi_{i}\right)
\end{array}\right]=0, \quad \text { for some } i \in\{1, \cdots, n\} .
$$

Note that there are altogether $C_{2}^{6}=30$ possible combinations of $k$ and $l$, where $k, l \in\{1, \cdots, 6\}$, and $k \neq l$. Now, let us denote $\operatorname{det}\left[\mathbf{b}_{k}^{T}\left(\phi_{i}, \psi_{i}\right), \mathbf{b}_{l}^{T}\left(\phi_{i}, \psi_{i}\right)\right]^{T}$ as $D_{k, l, i}$. Clearly, if $D_{k, l, i}=0$, then $D_{l, k, i}=-D_{k, l, i}=0$. Thus, we may consider just 15 possible combinations of $k$ and $l$, where $k, l \in\{1, \cdots, 6\}$, and $k<l$. In this connection, it can be verified that

$$
\begin{aligned}
& D_{1,2, i}=D_{4,5, i}=\sin \psi_{i}=0 \text { if and only if } \psi_{i}=0 \\
& D_{1,3, i}=D_{4,6, i}=-\sin \phi_{i} \cos \phi_{i}=0
\end{aligned}
$$$$
\text { if and only if } \phi_{i}=0, \pi \text { or } \psi_{i}= \pm \frac{\pi}{2}
$$$$
D_{1,4, i}=\left(-1+\cos ^{2} \phi_{i} \cos ^{2} \psi_{i}\right)=0
$$

if and only if $\phi_{i}=0, \pi$ and $\psi_{i}=0$

$D_{1,5, i}=D_{2,4, i}=\sin \phi_{i} \cos \phi_{i} \cos ^{2} \psi_{i}=0$ if and only if $\phi_{i}=-\frac{\pi}{2}, 0, \frac{\pi}{2}, \pi$ or $\psi_{i}= \pm \frac{\pi}{2}$

$D_{1,6, i}=D_{3,4, i}=\cos \phi_{i} \sin \psi_{i} \cos \psi_{i}=0$ if and only if $\phi_{i}= \pm \frac{\pi}{2}$ or $\psi_{i}=0, \pm \frac{\pi}{2}$;

$D_{2,3, i}=D_{5,6, i}=\cos \phi_{i} \cos \psi_{i}=0$ if and only if $\phi_{i}= \pm \frac{\pi}{2}$ or $\psi_{i}= \pm \frac{\pi}{2}$

$D_{2,5, i}=\left(-1+\sin ^{2} \phi_{i} \cos ^{2} \psi_{i}\right)=0$ if and only if $\phi_{i}= \pm \frac{\pi}{2}$ and $\psi_{i}=0$

$D_{2,6, i}=D_{3,5, i}=\sin \phi_{i} \sin \psi_{i} \cos \psi_{i}=0$ if and only if $\phi_{i}=0, \pi$ or $\psi_{i}=0, \pm \frac{\pi}{2}$

$D_{3,6, i}=-\cos ^{2} \psi_{i}=0$ if and only if $\psi_{i}= \pm \frac{\pi}{2}$.

Therefore, the value of $D_{k, l, i}$, where $l, k=1, \cdots, 6$, equals 0 only if $\phi_{i}=p \pi / 2$, where $p \in\{-1,0,1,2\}$ or $\psi_{i}=0$. This contradicts our hypothesis that Assumption 5 holds. In conclusion, the matrix $\left[\mathbf{A}_{k}^{T}, \mathbf{A}_{l}^{T}\right]^{T}$ is of full column rank if both Assumption 5 and Condition i) of Assumption 4 hold.

$$
\mathbf{N}_{k}=\left[\begin{array}{ccccc}
\mathbf{b}_{k}\left(\phi_{1}, \psi_{1}\right) & \mathbf{0}_{1 \times 2} & \cdots & \cdots & \mathbf{0}_{1 \times 2} \\
\mathbf{0}_{1 \times 2} & \mathbf{b}_{k}\left(\phi_{2}, \psi_{2}\right) & \mathbf{0}_{1 \times 2} & \cdots & \mathbf{0}_{1 \times 2} \\
\vdots & \mathbf{0}_{1 \times 2} & \ddots & & \vdots \\
\vdots & \vdots & & & \vdots \\
\mathbf{0}_{1 \times 2} & \mathbf{0}_{1 \times 2} & \cdots & & \mathbf{b}_{k}\left(\phi_{n}, \psi_{n}\right)
\end{array}\right] \in \mathbb{C}^{n \times 2 n}
$$




\section{APPENDIX C}

DERIVATION OF (27) AND (28)

Recall that in the first coordinate system, $\mathbf{v}_{1, k}$ and $\mathbf{v}_{2, k}$ are two orthogonal vectors that span the same plane as the electric and magnetic field vectors of the $k$ th signal. Now, let us denote, for the new coordinate system, $\hat{\mathbf{v}}_{1, k}$ and $\hat{\mathbf{v}}_{2, k}$ as the corresponding two orthogonal vectors that span the same plane as the electric and magnetic field vectors of the $k$ th signal. [Note that, in terms of $\hat{\phi}_{k}$ and $\hat{\psi}_{k}$, $\hat{\mathbf{v}}_{1, k}$ and $\hat{\mathbf{v}}_{2, k}$ can be expressed as $\left(\cos \hat{\phi}_{k} \quad 0-\sin \hat{\phi}_{k}\right)$ and $\left(-\sin \hat{\phi}_{k} \sin \hat{\psi}_{k} \cos \hat{\psi}_{k}-\cos \hat{\phi}_{k} \sin \hat{\psi}_{k}\right)$, respectively.] Thus, geometrically, the vectors $\mathbf{v}_{1, k}, \mathbf{v}_{2, k}, \hat{\mathbf{v}}_{1, k}$, and $\hat{\mathbf{v}}_{2, k}$ all lie on the same plane, and $\hat{\mathbf{v}}_{1, k}$ (or $\hat{\mathbf{v}}_{2, k}$ ) is the rotation of the vector $\mathbf{v}_{1, k}$ (or $\mathbf{v}_{2, k}$ ) via angle $\hat{\delta}_{k} \in(-\pi, \pi]$ in the plane containing $\mathbf{v}_{1, k}$ and $\mathbf{v}_{2, k}$. Thus, the vectors are related as

$$
\left(\begin{array}{ll}
\mathbf{v}_{1, k} & \mathbf{v}_{2, k}
\end{array}\right)=\left(\begin{array}{ll}
\hat{\mathbf{v}}_{1, k} & \hat{\mathbf{v}}_{2, k}
\end{array}\right) \mathbf{Q}\left(\hat{\delta}_{k}\right)
$$

where $\mathrm{Q}$ is the rotation matrix as defined in (6). Then, it follows from (9) and (40) that we can express the matrix $\mathbf{B}\left(\phi_{k}, \psi_{k}\right)$ in terms of $\hat{\phi}_{k}$ and $\hat{\psi}_{k}$ as

$$
\mathbf{B}\left(\phi_{k}, \psi_{k}\right)=\left(\begin{array}{rr}
\hat{\mathbf{v}}_{1, k} & \hat{\mathbf{v}}_{2, k} \\
\hat{\mathbf{v}}_{2, k} & -\hat{\mathbf{v}}_{1, k}
\end{array}\right) \mathbf{Q}\left(\hat{\delta}_{k}\right) .
$$

Now, let us denote

$$
\hat{\mathbf{T}}=\left[\begin{array}{cccc}
\mathbf{Q}\left(\hat{\delta}_{1}\right) & \mathbf{0}_{2 \times 2} & \cdots & \mathbf{0}_{2 \times 2} \\
\mathbf{0}_{2 \times 2} & \mathbf{Q}\left(\hat{\delta}_{2}\right) & & \mathbf{0}_{2 \times 2} \\
\vdots & & \ddots & \vdots \\
\mathbf{0}_{2 \times 2} & \mathbf{0}_{2 \times 2} & \cdots & \mathbf{Q}\left(\hat{\delta}_{n}\right)
\end{array}\right]
$$

where $0_{2 \times 2}$ denotes the $(2 \times 2)$ zero matrix. Then, it can be verified using (8) and (41) that

$$
\left(\begin{array}{l}
\mathbf{A}_{3} \\
\mathbf{A}_{1}
\end{array}\right) \hat{\mathbf{T}}^{-1}=\left(\begin{array}{r}
\mathbf{A}_{4} \\
-\mathbf{A}_{6}
\end{array}\right) \hat{\mathbf{T}}^{-1} \hat{\mathbf{D}}^{(1)}
$$

where

$$
\begin{aligned}
\hat{\mathrm{D}}^{(1)} & =\operatorname{diag}\left\{\hat{d}_{1}^{(1)}, \cdots, \hat{d}_{2 n}^{(1)}\right\} \\
\hat{d}_{2 i-1}^{(1)} & =\frac{1}{\sin \hat{\psi}_{i}} \\
\hat{d}_{2 i}^{(1)} & =\sin \hat{\psi}_{i}, \quad \text { for } i=1, \cdots, n .
\end{aligned}
$$

Moreover

$$
\left(\begin{array}{l}
\mathbf{A}_{3} \\
\mathbf{A}_{4}
\end{array}\right) \hat{\mathbf{T}}^{-1}=\left(\begin{array}{r}
-\mathbf{A}_{1} \\
\mathbf{A}_{6}
\end{array}\right) \hat{\mathbf{T}}^{-1} \hat{\mathbf{D}}^{(2)}
$$

where

$$
\begin{aligned}
\hat{\mathbf{D}}^{(2)} & =\operatorname{diag}\left\{\hat{d}_{1}^{(2)}, \cdots, \hat{d}_{2 n}^{(2)}\right\} \\
\hat{d}_{2 i-1}^{(2)} & =\tan \hat{\phi}_{i} \\
\hat{d}_{2 i}^{(2)} & =-\frac{1}{\tan \hat{\phi}_{i}}, \quad \text { for } i=1, \cdots, n .
\end{aligned}
$$

Equations (27) and (28) can then be obtained directly from (16), (42), and (43).

\section{ACKNOWLEDGMENT}

The authors are thankful to the reviewers for their insightful and useful comments/suggestions. In particular, the comments of one of the reviewers led to the idea presented in the paragraph before Section V-E.

\section{REFERENCES}

[1] A. Nehorai and E. Paldi, "Vector-sensor array processing for electromagnetic source localization," IEEE Trans. Signal Processing, vol. 42, pp. 376-398, Feb. 1994.

[2] K.-C. Tan, K.-C. Ho, and A. Nehorai, "Linear independence of steering vectors of an electromagnetic vector sensor," IEEE Trans. Signal Processing, vol. 44, pp. 3099-3107, Dec. 1996.

[3] K.-C. Ho, K.-C. Tan, and W. Ser, "An investigation on number of signals whose directions-of-arrival are uniquely determinable with an electromagnetic vector sensor," Signal Process., vol. 47, no. 1, pp. 41-54, Nov. 1995.

[4] B. Hochwald and A. Nehorai, "Identifiability in array processing models with vector-sensor applications," in Proc. 7th SP Workshop SSAP, June 1994, pp. 121-124.

[5] _ _ "Identifiability in array processing models with vector-sensor applications," IEEE Trans. Signal Processing, vol. 44, pp. 83-95, Jan. 1996.

[6] J. Li, "Direction and polarization estimation using arrays with small loops and short dipoles," IEEE Trans. Antennas Propagat., vol. 41, pp. 379-387, Mar. 1993.

[7] K. T. Wong and M. D. Zoltowski, "High accuracy 2D angle estimation with extended aperture vector sensor arrays," in Proc. ICASSP, Atlanta, GA, vol. 5, May 1996, pp. 2789-2792.

[8] K.-C. Tan and Z. Goh, "A detailed derivation of arrays free of higher rank ambiguities," IEEE Trans. Signal Processing, vol. 44, pp. 351-359, Feb. 1996.

[9] M. Skolnik, Radar Handbook, 2nd ed. New York: McGraw-Hill.

[10] J. Li and P. Stoica, "Efficient parameter estimation of partially polarized electromagnetic waves," IEEE Trans. Signal Processing, vol. 42, pp. 3114-3125, Nov 1994.

[11] K.-C. Ho, K.-C. Tan, and B. T. G. Tan, "Estimation of directionsof-arrival of partially polarized signals with electromagnetic vector sensors," Proc. ICASSP, May 1996, vol. 5, pp. 2900-2903.

[12] B. Hochwald and A. Nehorai, "Polarimetric modeling and parameter estimation with applications to remote sensing," IEEE Trans. Signal Processing, vol. 43, pp. 1923-1935, Aug. 1995.

[13] R. Roy and T. Kailath, "ESPRIT-Estimation of signal parameters via rotational invariance techniques," IEEE Trans. Acoust., Speech, Signal Processing, vol. 37, pp. 984-995, July 1989.

[14] K.-C. Tan, G.-L. Oh, and M. H. Er, "A study of the uniqueness of steering vectors in array processing," Signal Process., vol. 34, no. 3, pp. 245-256, Dec. 1993.

[15] R. O. Schmidt, "A signal subspace approach to multiple emitter location and spectral estimation," Ph.D. dissertation, Dept. Electr. Eng., Stanford Univ., Stanford, CA, 1981.

[16] M. Wax and T. Kailath, "Detection of signals by information theoretic criteria," IEEE Trans. Acoust., Speech, Signal Processing, vol. ASSP-33, pp. 387-392, Apr. 1985.

Kwok-Chiang Ho was born in Singapore in 1968. He received the B.Sc (Hons) degree in 1993 in mathematics from the National University of Singapore.

From 1993 to 1996, he worked at the Defence Science Organization of Ministry of Defence, Singapore, as an Analyst. Since August 1996, he has been working at the Centre for Signal Processing, Nanyang Technological University, as a Project Officer. His research interests are in array signal processing, digital communications, and speech processing. 
Kah-Chye Tan was born in Singapore in 1961. He received the B.Sc (Hons) degree and the Ph.D degree, both in physics and both from the National University of Singapore, in 1985 and 1992, respectively.

From 1985 to 1996, he worked at the Defence Science Organization of the Ministry of Defence, Singapore, as a Principal Analyst. Since August 1996, he has been working at the Centre for Signal Processing, Nanyang Technological University, Singapore, as a Research and Development Manager. His research interests are in array signal processing, speech/audio processing, image/video processing, and digital communications.

Dr. Tan received the Defence Technology Prize (individual) in 1993 and the Institute of Physics Medal in 1985.
B. T. G. Tan received the B.Sc. (Hons) degree in physics in 1965 from the University of Singapore, and the D.Phil. degree in 1968 from Oxford University, Oxford, U.K.

Since 1968, he has taught at the National University of Singapore, where he is now an Associate Professor in physics. His research interests include the microwave properties of semiconductors and dielectrics, surface physics, image processing, and digital sound synthesis.

Dr. Tan is a chartered engineer and a member of the Institution of Electrical Engineers. 